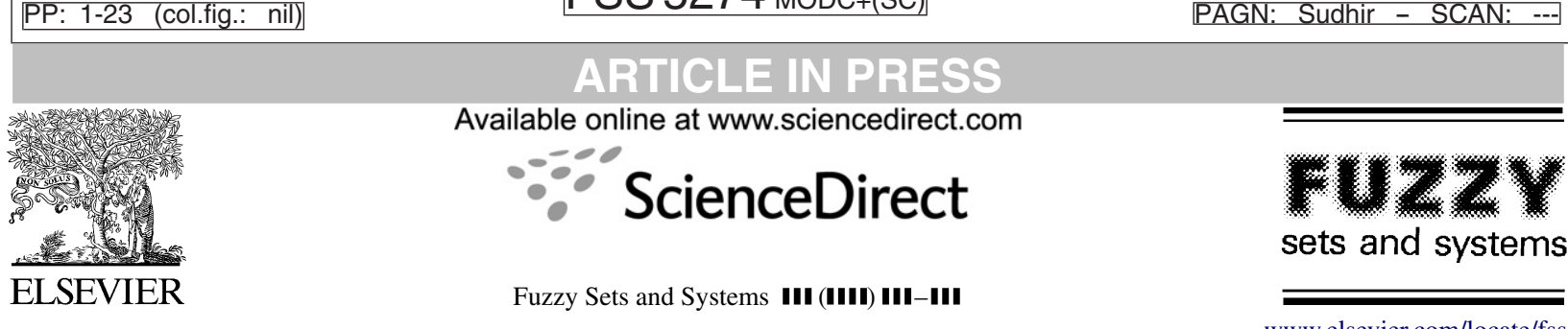

www.elsevier.com/locate/fss

\title{
On the granularity of summative kernels
}

\author{
Kevin Loquin*, Olivier Strauss
}

Laboratoire d'Informatique, de Robotique et de Microélectronique de Montpellier, Université Montpellier II, 161, rue Ada, 34392 Montpellier

\author{
Cedex 5, France
}

\section{Abstract}

In this paper, we propose granularity as a new index to characterize the non-specificity of a summative kernel. This index is intended to reflect the behavior of a kernel in the usual signal processing applications. We show, in different experiments, that two kernels having the same granularity have very similar behavior. This granularity-based adaptation is compared to other adaptation methods. These experiments highlight the ability of the granularity index to measure the spreading and collecting properties of a summative kernel.

(c) 2008 Elsevier B.V. All rights reserved.

\section{Introduction}

A wide range of digital analysis and signal processing [16] procedures inherently rely on methods for reconstructing a continuous underlying signal from a set of sampled corrupted values. These values are usually uniformly sampled, since the measures come from systematic observations. Kernels are essential tools in this context, since they are used in reconstruction, impulse response modeling, resampling, interpolation, linear or non-linear transformations, stochastic or band-pass filtering, etc. In digital signal processing, kernels are mainly used to derive discrete algorithms from a continuous representation. Within most applications, a kernel can be seen as a weighted neighborhood ensuring a smooth interplay between continuous and discrete domains. They can be visualized as bumps that can be shifted to any location of the signal domain, so as to absorb or spread the information contained in the signal. They are often bounded, monomodal and symmetric. In this paper, the kernels will be defined on a domain $\Omega$, subset of $\mathbb{R}^{p}$, for $p \in \mathbb{N}$.

Digital signal derivation $[15,3,5]$ is a typical example of such an application. The classical finite differences method usually fails to perform the estimation of the derivative of the signal, especially with a noisy signal. The kernel-based method consists of computing the sampled derivative of an estimation of the continuous signal. This estimation is obtained by convolving the original discrete signal with a continuous kernel, chosen to lower the impact of both acquisition noise and quantization effect. The implementation of such a method simply consists of convolving the original sampled signal with the derivative of the chosen kernel, which is also a kernel.

Most of the kernels used in signal processing are summative kernels, or linear combinations of summative kernels. A summative kernel is a positive function, the integral of which equals 1. For instance, splines [32] are summative kernels, the derivatives of which are linear combinations of splines.

\footnotetext{
* Corresponding author. Tel.: +3346741 85 65; fax: +33467418500.

E-mail addresses: kevin.loquin@lirmm.fr (K. Loquin), olivier.strauss@lirmm.fr (O. Strauss).
} 
One problem of practical importance is the characterization of the behavior of a summative kernel in a particular application. Our aim, in this paper, is to define an index reflecting the ability of a summative kernel to absorb or spread

\section{the information contained in the signal. This is particularly sensible when the sumative kernel is used to model the} impulse response of a sensor. In that case, this index should capture the non-resolution power of the sensor, i.e. its power of information collecting. In this paper, we generalize the use of the expression non-resolution power to any summative kernel, and not only those modeling a sensor, as being its ability to collect or spread information. Note that, since the neighborhood, represented by the kernel, can be shifted to different locations of the signal, this non-resolution power should not depend on its location. When considering a simple crisp neighborhood, its non-resolution power can

9 be naturally quantified by its Lebesgue measure, which is a continuous generalization of the concept of cardinality of a discrete set. This quantification of non-resolution power of a crisp neighborhood can easily be generalized to

11 fuzzy neighborhoods by extending the concept of cardinality to the continuous domain. Cardinality is an index of the non-specificity of a fuzzy neighborhood. Specificity, as stated in [8,35], can be considered as a distance measure

13 between the fuzzy neighborhood considered and a fuzzy set that contains only one element. It can be reformulated in the following manner: the specificity of a fuzzy neighborhood is its ability to be concentrated on a set of minimal length (in the sense of the Lebesgue measure of this set), i.e. its ability to be influent on specific parts of the domain of the neighborhood. However, no such index has been defined for a summative kernel-based weighted neighborhood.

17 Since a summative kernel, as defined above, can also be seen as a probability distribution, it seems natural to consider existing information measures of probability distribution, regarding their relevance as indices of non-resolution power.

19 The notion of informativity, for a probability distribution, corresponds to the information needed to construct it. In the literature, an information measure of a probability distribution is supposed to quantify its informativity. This

21 quantification is however sometimes doubtful, since what is captured by an information measure of a probability distribution is, rather, the geometrical properties of the distribution, such as its flatness, its peakedness, or its average difference from the mean. To take a simple example: uniform distribution, whether obtained under full knowledge (i.e. where it is known that every alternative of $\Omega$ has the same probability of occurring) or under total indeterminacy and by the use of the principle of insufficient reason (i.e. where nothing is known about the probabilities of the alternatives of $\Omega$ ), will have the same shape and the same information measure, whereas the informativity is different.

In this paper, we propose a non-resolution power index for a summative kernel-based weighted neighborhood under the name of granularity, by reference to the works of Pawlak [21], who defined the granularity of a rough set as its power of resolution, and to the more recent works of Bodjanova [2], who defined a concept of granulation for a fuzzy set.

31 This paper is organized as follows. After this introduction, Section 2 presents the concepts of maxitive and summative kernels. Each kernel is associated with a model of uncertainty: possibility and probability measures, respectively.

33 Considering each model, we present different ways of defining the non-specificity of a weighted neighborhood. Section 3 is dedicated to the construction of granularity as an index of non-specificity. Section 4 presents a granularity-based

35 method for adapting kernels. This method is compared to other adaptation methods. In Section 5 we expose illustrative experiments highlighting the ability of granularity to measure the spreading and collecting properties of a summative 37 kernel.

\section{Kernels, uncertainty and information}

\subsection{Maxitive kernels, possibility distributions and normalized fuzzy subsets}

What will be called a maxitive kernel in this paper is a possibility distribution or membership function of a normalized fuzzy subset [36]. It can be considered as a weighted neighborhood, dispersing or collecting information around a given location, called the mode. The mode can be a singleton or a set. Furthermore, the extent of this neighborhood is delimited by its support.

Definition 1. A maxitive kernel is a $[0,1]$-valued function $\pi$, defined on a domain $\Omega$, verifying the maxitivity property

$$
\sup _{\omega \in \Omega} \pi(\omega)=1
$$

Its mode is given by $\operatorname{Mode}(\pi)=\{\omega \in \Omega / \pi(\omega)=1\}$ and its support is $\operatorname{Supp}(\pi)=\{\omega \in \Omega / \pi(\omega)>0\}$. 

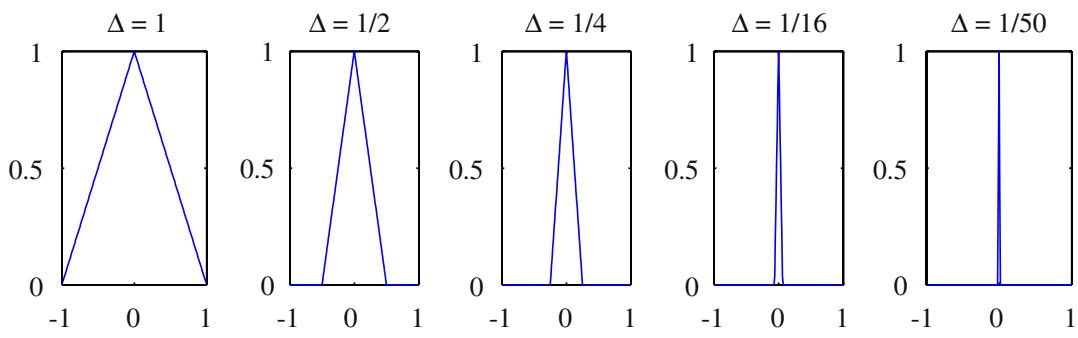

Fig. 1. Triangular family of maxitive kernels.

1 Note that any given monomodal maxitive kernel $\pi$, defined on $\Omega$, can be the basis for a family of maxitive kernels tuned by a location-scale parameter $\theta=(m, \Delta)$, with $m$ a translation factor and $\Delta$ its bandwidth. Any element of this family is obtained, for $m \in \Omega$ and $\Delta>0$, by

$$
\pi_{\Delta}^{m}(\omega)=\pi\left(\frac{\omega-m}{\Delta}\right), \quad \forall \omega \in \Omega
$$

5 Fig. 1 consists of five maxitive kernels $\pi_{\Lambda}^{0}$ of the family that has for its basic maxitive kernel the triangular kernel $\pi(\omega)=(1-|\omega|) \mathbb{1}_{[-1,1]}(\omega)$.

$7 \quad$ Note that Fig. 1 is in agreement with Theorem 2, which tells us that the Kronecker function $\delta^{m}$ is the limit of a sequence of maxitive distributions whose graphs become thin and peak around $\omega=m$. The Kronecker function is defined by $\delta^{m}(\omega)=1$ for $\omega=m$ and 0 otherwise. The convergence is a modified uniform convergence, similar to the convergence in distributions (see [26] and Theorem 5). Indeed it also involves a product of functions, but here the sup replaces the integral of the convergence in distributions.

Theorem 2. For a fixed $m \in \Omega\left(\subseteq \mathbb{R}^{p}\right)$, let $\pi_{\Delta}^{m}$ be monomodal maxitive kernels on $\Omega$, with mode $m$, such that for any $a>0, \sup _{B_{a}} \pi_{\Delta}^{m}(\omega) \rightarrow 0$ as $\Delta \rightarrow 0$, with $B_{a}=\left\{\omega \in \Omega / \forall i \in\{1, \ldots, p\},\left|\omega_{i}-m_{i}\right| \geqslant a\right\}$, then $\pi_{\Delta}^{m} \longrightarrow \delta^{m}$, as $\Delta-$ $\rightarrow 0$, in the sense of a modified uniform convergence, i.e. $\lim _{\Delta \rightarrow 0} \sup _{\Omega}\left(\pi_{\Delta}^{m}(\omega) \varphi(\omega)\right)=\sup _{\Omega}\left(\delta^{m}(\omega) \varphi(\omega)\right)=\varphi(m)$, whatever $\varphi$ a positive function.

Proof 3. We note $A=\lim _{\Delta \rightarrow 0} \sup _{\Omega}\left(\pi_{\Delta}^{m}(\omega) \varphi(\omega)\right)$. First, since $\Omega=\left(\cup_{a>0} B_{a}\right) \cup\{m\}$, then $A=\lim _{\Delta \rightarrow 0} \sup _{\left(\cup_{a>0} B_{a}\right) \cup\{m\}}$ $\left(\pi_{\Delta}^{m}(\omega) \varphi(\omega)\right)$. Since for any $a>0, \lim _{\Delta \rightarrow 0} \sup _{B_{a}} \pi_{\Delta}^{m}(\omega)=0$, the sup of the last expression is obtained for $\omega=m$, therefore, $A=\lim _{\Delta \rightarrow 0} \pi_{\Delta}^{m}(m) \varphi(m)=\varphi(m)$, because whatever $m$ and $\Delta, \pi_{\Delta}^{m}(m)=1$. Then, it is sufficient to observe that $\varphi(m)=\sup _{\Omega}\left(\delta^{m}(\omega) \varphi(\omega)\right)$, which is true with $\varphi \geqslant 0$.

A possibility distribution $\pi$ has a relevant meaning in the scope of uncertainty theories. $\pi$ induces a possibility measure, noted $\Pi$, computed in this way:

$$
\forall A \subseteq \Omega, \quad \Pi(A)=\sup _{\omega \in A} \pi(\omega)
$$

which verifies the maxitivity axiom for possibility distributions defined on an infinite domain [19]:

$$
\forall\left(A_{n}\right)_{n>0} \subseteq \Omega, \quad \Pi\left(\bigcup_{n>0} A_{n}\right)=\max _{n>0}\left(\Pi\left(A_{n}\right)\right) .
$$

The value $\Pi(A)$ can be interpreted as a degree of possibility for a realization of the underlying uncertain phenomenon to fall in $A$. As stated in $[34,4,9]$, a possibility measure is one particular case of upper probability, a notion introduced by Walley [33]. Note that we can obtain the possibility distribution $\pi$ from its measure $\Pi$ on singletons by $\pi(\omega)=\Pi(\{\omega\})$, $\forall \omega \in \Omega$. 


\subsection{Construction of possibility distributions from orderings}

The notion of degree is parallel to the notion of ordering. Indeed, every element, whose degree of appropriateness to a concept is given, can be ordered in a numerical scale. Conversely, every concept, according to which elements may be ordered, induces degrees of appropriateness of these elements to this concept. To take the example of mammals, an elephant can be thought of as being more a mammal than a duck-billed platypus, because the latter lays eggs, which is not a feature of mammals. When the underlying concept depends on numerical values, as for instance the concept of "tall" depends on height, it is more sensible and natural to build a numerical scale of degrees from the ordering induced by the heights.

9 Therefore, any ordering on alternatives (the $\omega$ of $\Omega$ ) numerically scaled by non-normalized degrees $d=(d(\omega))_{\omega \in \Omega}$ can induce a fuzzy subset, whose membership function is a set of degrees on the alternatives. To comply with possibility

11 theory, these non-normalized degrees $d$ induce a set function $D(A)=\sup _{\omega \in A} d(\omega), \forall A \subseteq \Omega$, which is not normalized either. For consistency of this ordering approach with the possibilistic interpretation of $D$, it is natural to assume that the degree of possibility that any realization of the uncertain phenomenon will fall in $\Omega$ is 1, i.e. that the post-normalized possibility measure, noted $\Pi$, fulfils the maxitivity property (1), which is equivalent to $\Pi(\Omega)=1$. A simple procedure

15 for achieving this normalization consists of dividing all the degrees $d$ by $D(\Omega)$, given by $D(\Omega)=\sup _{\omega \in \Omega} d(\omega)$. The post-normalized degree of possibility is $\pi(\omega)=d(\omega) / D(\Omega), \forall \omega \in \Omega$. This normalization is also consistent when

17 interpreting $\pi$ as a maxitive kernel, i.e. the weighted neighborhood of a location. Indeed, the mode is a set of locations fully in accordance with the concept represented by the neighborhood.

19 In a more formal way, for a set of alternatives $\omega \in \Omega$, a preference ordering $\succcurlyeq_{\pi}$ is equivalent to a possibility distribution $\pi$ by

$$
\omega_{1} \succcurlyeq_{\pi} \omega_{2} \Longleftrightarrow \pi\left(\omega_{1}\right) \geqslant \pi\left(\omega_{2}\right) .
$$

Preferring one alternative to another is equivalent to saying that one alternative is more possible than another.

\subsection{Possibility and non-specificity}

First, the concept of specificity already exposed in the literature $[35,8]$ of fuzzy sets and possibility theories should be remembered. Specificity is the ability for a fuzzy neighborhood to be concentrated on a set of minimal length (in the sense of the Lebesgue measure of this set). Measures of specificity effectively capture the idea of how close a fuzzy set is to a singleton. The Kronecker function at any given location $\omega$ of $\Omega$ is the most specific possibility distribution, whereas the vacuous possibility distribution on $\Omega$, which equals 1 on $\Omega$, is the least specific distribution. The first part of this remark, associated with Theorem 2 , leads to noting that, for a family of maxitive kernels $\left(\pi_{\Delta}^{m}\right)_{\Delta>0}$, given for any $m \in \Omega$, the specificity of $\pi_{\Delta}^{m}$ increases when $\Delta \longrightarrow 0$, since this family tends to the Kronecker function.

The continuous generalization of the cardinality of a fuzzy subset $F$, whose membership function is $\mu_{F}(\omega)=\pi(\omega)$, $\forall \omega$, is defined by the following expression:

$$
\operatorname{card}(\pi)=\int_{\Omega} \pi(\omega) \mathrm{d} \omega
$$

This is a natural measure of its non-specificity.

Note that when $\pi$ is interpreted as a maxitive kernel, its cardinality is a natural measure of its non-resolution power.

\subsection{Summative kernels and probability distributions}

What is called a summative kernel, in this paper, is a probability distribution. As for a maxitive kernel, it can be considered as a weighted neighborhood, dispersing or collecting information around a given location, called the mode.

The mode can be a singleton or a set. Furthermore, the extent of this neighborhood is delimited by its support.

Definition 4. Summative kernels are $\mathbb{R}^{+}$-valued functions $\kappa$ defined on a domain $\Omega$, verifying the summativity property

$$
\int_{\Omega} \kappa(x) \mathrm{d} x=1
$$



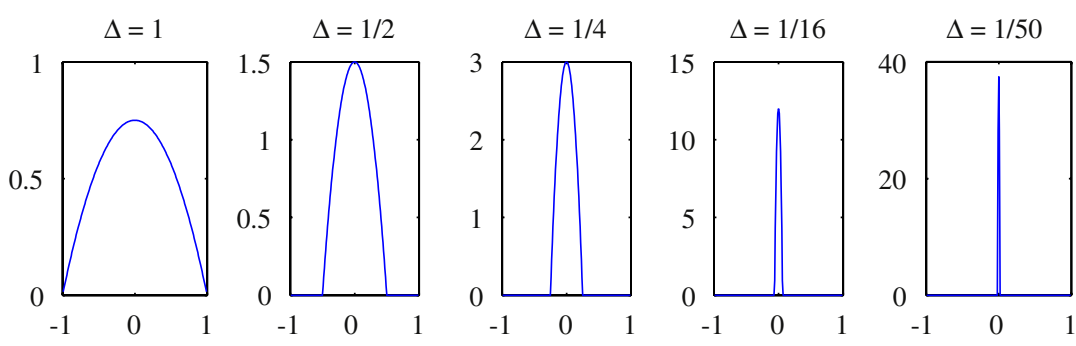

Fig. 2. Epanechnikov family of summative kernels.

1 The mode of a summative kernel $\kappa$, noted $\operatorname{Mode}(\kappa)$, are the values $\omega$ at which $\kappa$ attains its maximum value. Its support is given by $\operatorname{Supp}(\kappa)=\{\omega \in \Omega / \kappa(\omega)>0\}$.

3 Note that any given monomodal summative kernel $\kappa$, can be the basis for a family of summative kernels tuned by a location-scale parameter $\theta=(m, \Delta)$, with $m$ a translation factor and $\Delta>0$ its bandwidth. Any element of this family

5 is obtained by

$$
\kappa_{\Delta}^{m}(\omega)=\frac{1}{\Delta} \kappa\left(\frac{\omega-m}{\Delta}\right), \quad \forall \omega \in \Omega
$$

7 Fig. 2 consists of five summative kernels $\kappa_{\Delta}^{0}$ of the family that has for its basic kernel the Epanechnikov kernel $\kappa(\omega)=\frac{3}{4}\left(1-\omega^{2}\right) 1_{[-1,1]}(\omega)$.

9 Note that Fig. 2 is in agreement with Theorem 5 (see [26]) which tells us that the Dirac delta $\delta^{m}$ (which is a distribution [26]) is the limit, in the sense of distributions, of a sequence of functions whose graphs become thin and tall, peaking around $\omega=m$. The Dirac delta, often referred to as the unit impulse function, has the value of $+\infty$ for $\omega=m$ and the value 0 elsewhere.

13 Theorem 5. For a fixed $m \in \Omega\left(\subseteq \mathbb{R}^{p}\right)$, let $\kappa_{\Delta}^{m}$ be monomodal summative kernels on $\Omega$, with mode m, such that for any $a>0, \int_{B_{a}} \kappa_{\Delta}^{m}(\omega) \mathrm{d} \omega \rightarrow 0$ as $\Delta \rightarrow 0$, with $B_{a}=\left\{\omega \in \Omega / \forall i \in\{1, \ldots, p\},\left|\omega_{i}-m_{i}\right| \geqslant a\right\}$, then $\kappa_{\Delta}^{m} \longrightarrow \delta^{m}$, as $\Delta \rightarrow 0$ in the sense of
positive test function.

17 As a probability distribution, $\kappa$ has a relevant meaning in the scope of uncertainty theories. It induces a probability measure, noted $P$, computed in this way

$$
\forall A \subset \Omega, \quad P(A)=\int_{A} \kappa(\omega) \mathrm{d} \omega,
$$

which verifies the Kolmogorov additivity axiom, which says that for any countable sequence of pairwise disjoint events $\left(A_{n}\right)_{n>0} \subseteq \Omega$,

$$
P\left(\bigcup_{n>0} A_{n}\right)=\sum_{n>0} P\left(A_{n}\right) .
$$

23 The value $P(A)$ can be interpreted as a degree of probability for a realization of the underlying uncertain phenomenon to fall in $A$.

\subsection{Construction of probability distributions from orderings}

In the same way that a possibility distribution $\pi$ can be obtained from a preference ordering $\succcurlyeq_{\pi}$ on the alternatives, a probability distribution $\kappa$ can be obtained from a preference ordering $\succcurlyeq_{\kappa}$ on the alternatives. In this sense, preferring one alternative to another is equivalent to saying that one alternative is more probable than another. 
To comply with probability theory, the non-normalized degrees, noted $d=(d(\omega))_{\omega \in \Omega}$, as in Section 2.2, induce, under the condition that $d \in \mathrm{七}_{1}$, a set function $D(A)=\int_{A} d(\omega) \mathrm{d} \omega, \forall A \subseteq \Omega$, which is not normalized either. The normalization procedure is different from the possibilistic, because of the summativity property (7). For consistency of this preference ordering approach with the probabilistic interpretation of $D$, it is natural to assume that the degree of probability that any realization of the uncertain phenomenon will fall in $\Omega$ is 1 , i.e. that the post-normalized probability measure, noted $P$, fulfils the summativity property (7), which is equivalent to $P(\Omega)=1$. A simple procedure for achieving this normalization consists of dividing all the degrees $d$ by $D(\Omega)$, given by $D(\Omega)=\int_{\Omega} d(\omega) \mathrm{d} \omega$. The post-normalization degree of probability is $\kappa(\omega)=d(\omega) / D(\Omega), \forall \omega \in \Omega$.

9 In a more formal way, for a set of alternatives $\omega \in \Omega$, a preference ordering $\succcurlyeq_{\kappa}$ is equivalent to a probability distribution $\kappa$ by

$$
\omega_{1} \succcurlyeq_{\kappa} \omega_{2} \Longleftrightarrow \kappa\left(\omega_{1}\right) \geqslant \kappa\left(\omega_{2}\right) .
$$

The preference ordering $\succcurlyeq_{\kappa}$ based on the probability distribution $\kappa$ has not the same meaning as the preference ordering $\succcurlyeq_{\pi}$ based on the possibility distribution $\pi$. It is the same as saying that a probability distribution is not the same as a possibility distribution [10]. Their semantics are different. Therefore, passing from one model (or one ordering) to another via their respective normalization is not sensible. In other words, it is not meaningful to build a possibility distribution from a probabilistic preference ordering; nor, conversely, to build a probability distribution from a possibilistic preference ordering.

\subsection{Probability and non-specificity}

First, the concept of specificity for a probability distribution is close to the specificity concept for a possibility distribution (see Section 2.3). Specificity is the ability, for a summative kernel or a probability distribution, to be concentrated on a set of minimal length (in the sense of the Lebesgue measure of this set). Measures of specificity should effectively capture the idea of how close a summative kernel is to a Dirac delta. The Dirac delta, at any given location $\omega$ of $\Omega$, is the most specific probability distribution, whereas the uniform probability distribution on $\Omega$, which equals $1 / \lambda(\Omega)$ on $\Omega$, should be the least specific distribution. $\lambda$ is the Lebesgue measure defined on the Borel subsets of $\Omega$. The first part of this remark, associated with Theorem 5, leads to noting that, for a family of summative kernels $\left(\kappa_{\Delta}^{m}\right)_{\Delta>0}$, given for any $m \in \Omega$, the specificity of $\kappa_{\Delta}^{m}$ increases when $\Delta \longrightarrow 0$, since this family tends to the Dirac delta.

27 We aim at defining, for summative kernels, an index of non-specificity reflecting its non-resolution power, in a way that is as natural as cardinality is an index of non-specificity for a maxitive kernel. As mentioned in the introductory part of this paper, no such index has been defined in this manner in the literature. However, since a summative kernel is a probability distribution, classical probability distribution information indices could be used as non-specificity indices. In 1948, Shannon introduced a measure of information for probability distribution known as ent
a probability distribution $\kappa$ defined on a infinite set of alternatives $\Omega$, the Shannon entropy is defined by

$$
H(\kappa)=-\int_{\Omega} \kappa(\omega) \log (\kappa(\omega)) \mathrm{d} \omega
$$

with the convention $0 \log 0:=0$.

This index is the unique solution of a set of axioms that may be found in [17], including the axioms of subadditivity and expansibility. The axiom of sub-additivity says that the amount of information in a joint probability distribution cannot be greater than the sum of the amounts of information in the associated marginal probabilities. The expansibility axiom claims that expanding the set of alternatives with another alternative not supported by evidence, i.e. with probability 0 , must not affect the amount of information. Rényi $[23,24]$ was probably the first to consider natural modifications in postulates of Shannon leading to non-Shannon entropies. The paper of Morales et al. [18] is a very useful survey of such non-Shannon indices.

Sometimes the variance of (a random variable associated with) a probability distribution is also considered as a measure of non-specificity. Variance, which measures the average distance between observations of the underlying uncertain phenomenon, is an index of dispersion for potential observations. For a probability distribution $\kappa$ defined on 

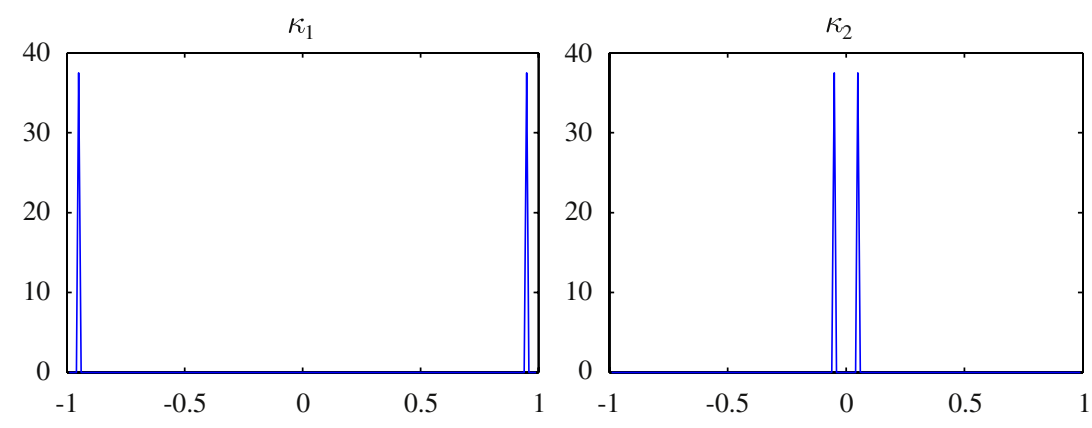

Fig. 3. Probability distributions with same specificity.

1 a infinite set of alternatives $\Omega$, the variance is defined by

$$
V(\kappa)=\int_{\Omega} \omega^{2} \kappa(\omega) \mathrm{d} \omega-\left(\int_{\Omega} \omega \kappa(\omega) \mathrm{d} \omega\right)^{2} .
$$

3 Here is a simple example to illustrate the fact that the variance does not measure the specificity.

Fig. 3 consists of two summative kernels, or probability distributions, $\kappa_{1}$ and $\kappa_{2}$. Each one is a mixture of two Epanechnikov distributions with $\Delta=0.01$ defining the same weighted neighborhoods around two modes. For $\kappa_{1}$, the modes are $\{-0.95,0.95\}$. For $\kappa_{2}$, the modes are $\{-0.05,0.05\}$. Therefore, both $\kappa_{1}$ and $\kappa_{2}$ have concentration sets with the same length, since they both peak around two modes in the same way. Thus, they should have the same specificity index. The variance of $\kappa_{1}$ being greater than the variance of $\kappa_{2}$, variance cannot be considered as a good specificity index. Actually, variance measures the dispersion of potential observations of probability distributions.

\section{Granularity}

\subsection{Natural ordering of the alternatives based on confidence intervals}

In the frequentist interpretation of probability, a confidence interval with confidence level $\alpha$ is an event (or a measurable set) of $\Omega$ such that the degree of probability, for any realization of the underlying uncertain phenomenon to fall in it, equals $\alpha$. In the framework of a subjective interpretation of probabilities, these intervals are named credible intervals. It is the key of the non-specificity index that we propose to capture the non-resolution power of a summative kernel.

The specificity of a summative kernel can be locally observed for a given degree. Fig. 4 consists of two summative kernels $\kappa_{1}$ and $\kappa_{2}$, qualitatively drawn, such that $\kappa_{2}$ is more specific than $\kappa_{1}$. For a given level $\alpha$, there are many confidence intervals. So as to compare the local specificity of two probability distributions, the smallest $\alpha$ confidence interval, in the sense of its Lebesgue measure, should be considered. This is called the most specific $\alpha$ confidence interval. It is $A$ for $\kappa_{1}$ and $B$ for $\kappa_{2}$. Since $\lambda(B)<\lambda(A)$, it seems natural to observe the specificity of a summative kernel, for a degree $\alpha$, by looking at the length of its most specific $\alpha$ confidence interval. Therefore, a global index of specificity should aggregate the observed specificities over the degrees $\alpha$.

In order to define this global specificity index, it will be easier to look at these confidence intervals in another direction, i.e. not by considering confidence intervals ranged by confidence level, but by looking at confidence intervals obtained from the elements $\omega$ of $\Omega$. They are defined, for all $\omega$ of $\Omega$, by

$$
I_{\omega}=\{x \in \Omega / \kappa(x) \geqslant \kappa(\omega)\} .
$$

$I_{\omega}$ is a confidence interval with confidence level $P\left(I_{\omega}\right)$. The set of all intervals $\left(I_{\omega}\right)_{\omega \in \Omega}$ enables an ordering on the alternatives $\omega$ of $\Omega$ by significance, weight and contribution to measurement of the specificity of $\kappa$. Indeed, the more specific $I_{\omega}$ is (i.e. the smallest with respect to the Lebesgue measure), the more significant $\omega$ is in quantifying the specificity of $\kappa$. Fig. 5 qualitatively illustrates this proposition. Each figure shows two confidence intervals, $I_{\omega_{1}}$ and $I_{\omega_{2}}$, of the summative kernel $\kappa$ seen as a probability distribution. $I_{\omega_{2}}$ is more specific than $I_{\omega_{1}}$, therefore $\omega_{2}$ should be more significant in the computation of the specificity index of $\kappa$ than $\omega_{1}$. 


\section{ARTICLE IN PRESS}

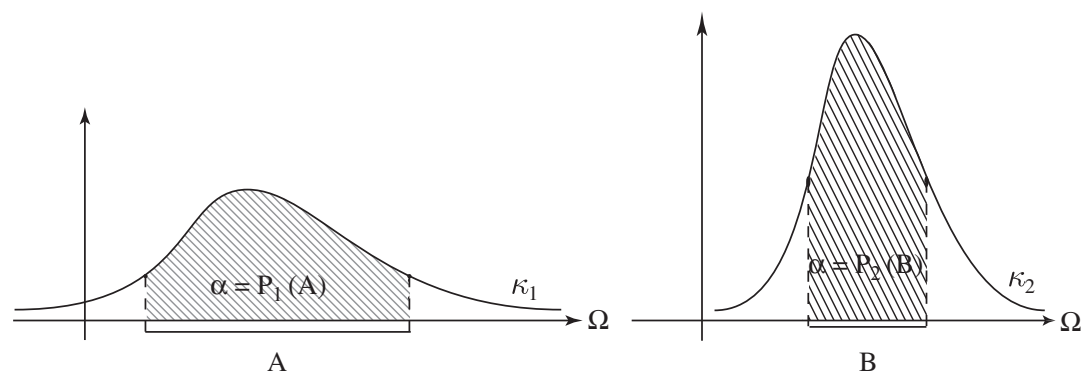

Fig. 4. Local specificity comparison between $\kappa_{1}$ and $\kappa_{2}$.
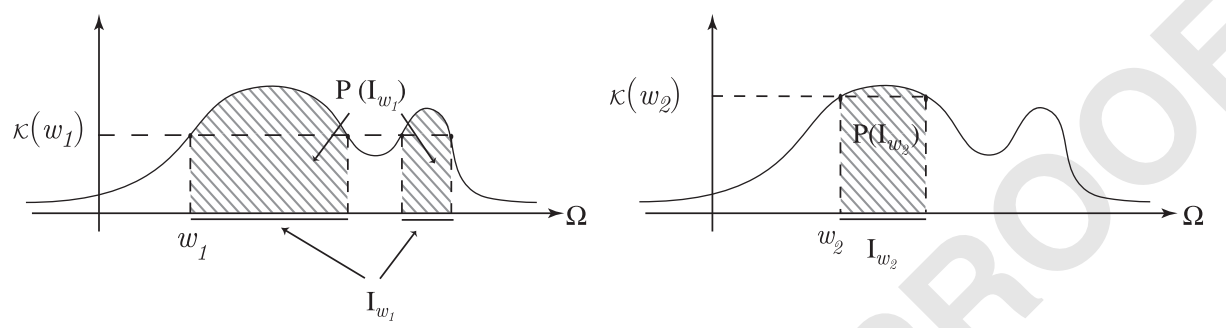

Fig. 5. Specificity contribution of $\omega_{1}$ and $\omega_{2}$.

Note that $\left(I_{\omega}\right)_{\omega \in \Omega}$ are nested sets. Indeed, for any $\omega_{1}$ and $\omega_{2}$ of $\Omega$, such that $\kappa\left(\omega_{1}\right) \geqslant \kappa\left(\omega_{2}\right)$, we have $I_{\omega_{1}} \subseteq I_{\omega_{2}}$, by definition (14). Therefore, if $I_{\omega_{1}}$ is more specific than $I_{\omega_{2}}$, then $\lambda\left(I_{\omega_{1}}\right)<\lambda\left(I_{\omega_{2}}\right)$, then $I_{\omega_{1}} \subseteq I_{\omega_{2}}$, and therefore $P\left(I_{\omega_{1}}\right)<P\left(I_{\omega_{2}}\right)$. This remark leads to a natural preorder on the alternatives $\omega$ of $\Omega$, according to their significance, for computing a specificity index of $\kappa$. This ordering, that we will note $\preccurlyeq_{\text {sp }}$, is given by

$$
\omega_{1} \preccurlyeq \mathrm{sp} \omega_{2} \Longleftrightarrow P\left(I_{\omega_{1}}\right) \geqslant P\left(I_{\omega_{2}}\right) .
$$

It is equivalent to

$$
\omega_{1} \preccurlyeq{ }_{\mathrm{sp}} \omega_{2} \Longleftrightarrow 1-P\left(I_{\omega_{1}}\right) \leqslant 1-P\left(I_{\omega_{2}}\right) .
$$

The $\preccurlyeq_{\text {sp }}$ preorder on the alternatives of $\Omega$ is based on probability distribution $\kappa$ but is different from $\preccurlyeq_{\kappa}$. Coupled naturally leads to the construction of a possibility distribution $\pi_{\leftarrow \kappa}$ from $\kappa$, which has for possibility degrees:

$$
\forall \omega \in \Omega, \quad \pi_{\leftarrow \kappa}(\omega)=1-P\left(I_{\omega}\right),
$$

since $1-P\left(I_{\omega}\right) \in[0,1], \forall \omega \in \Omega$. In a more formal way, $\preccurlyeq_{\mathrm{sp}} \Leftrightarrow \preccurlyeq_{\leftarrow \kappa}$. This construction of a possibility distribution $\pi_{\leftarrow \kappa}$, instead of a probability distribution, is natural, since the normalization is made in the construction. Indeed $\pi_{\leftarrow \kappa}(\omega)=1$ when $\omega \in \operatorname{Mode}(\kappa)$.

15 Obtaining this possibility distribution $\pi_{\leftarrow \kappa}$ is the first step for defining granularity of a summative kernel. $\pi_{\leftarrow \kappa}$ is the distribution which rearranges information concerning the specificity weights of the alternatives of $\Omega$ in an ordered way. $\pi_{\leftarrow \kappa}$ will be called the possibility distribution for specificity of $\kappa$.

Note that the possibility distribution obtained is exactly the solution of the probability/possibility transformation of Dubois and Prade [12,6,11].

\subsection{Probability/possibility transformations}

21 Dubois et al. have proposed three principles to be fulfilled by a sensible probability/possibility transformation: consistency, preservation of the preference ordering and maximal specificity. 


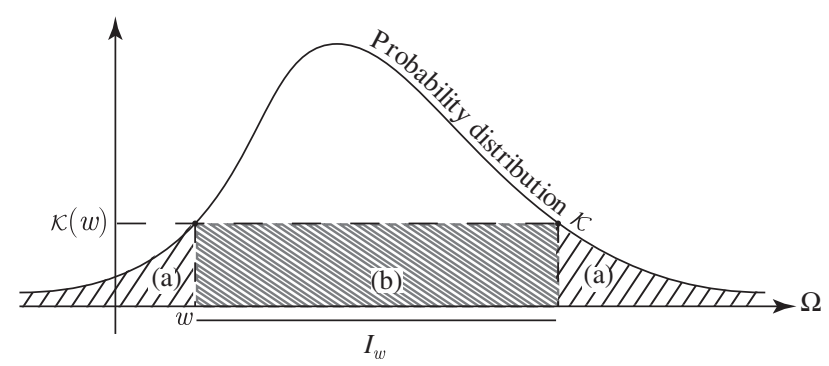

Fig. 6. Illustration of transformations $\pi_{[\kappa]}(\omega)$ and $\pi_{\leftarrow \kappa}(\omega)$.

Consistency between transformation components is an obvious first requirement for proper definitions of transformations between a probability distribution $\kappa$ and a possibility distribution $\pi$. It postulates that any event $A \subseteq \Omega$ must have a higher degree of possibility than degree of probability, i.e. $\Pi(A) \geqslant P(A)$. It was first termed consistency by Zadeh [36], and is close to the notion of coherence in the imprecise probability theory of Walley, where the domain $\Omega$ is discrete.

The preservation of the preference ordering can be defined in this way: an element $\omega_{1}$ preferred to an element $\omega_{2}$ in probability should be preferred in possibility. Formally, $\kappa\left(\omega_{1}\right)>\kappa\left(\omega_{2}\right) \Rightarrow \pi\left(\omega_{1}\right)>\pi\left(\omega_{2}\right)$ or $\omega_{1} \succ_{\kappa} \omega_{2} \Rightarrow \omega_{1} \succ_{\pi}$ $\omega_{2}$, when $\kappa$ and $\pi$ are respectively the probability and the possibility distribution associated with $P$ and $\Pi$. Within this principle, equally probable elements do not need to be equally possible. Note that $\kappa\left(\omega_{1}\right)>\kappa\left(\omega_{2}\right) \Rightarrow I_{\omega_{1}} \subset I_{\omega_{2}} \Rightarrow$ $1-P\left(I_{\omega_{1}}\right)>1-P\left(I_{\omega_{2}}\right) \Rightarrow \pi_{\leftarrow \kappa}\left(\omega_{1}\right)>\pi_{\leftarrow \kappa}\left(\omega_{2}\right)$.

When only the two previous principles are fulfilled, the transformation results in a set of possibility distributions instead of a unique possibility distribution.

Maximal specificity results in the choice of the possibility distribution having minimal cardinality. The possibility distribution fulfilling these principles is exactly the possibility distribution for specificity of $\kappa$, given by expression (16).

Dubois [6], proposed another transformation, that we note $\pi_{[\kappa]}$ and which is called the subjective transformation, because it is generally preferred to $\pi_{\leftarrow \kappa}$ in a subjective context. This is the converse transformation of the pignistic transformation defined by Smets [31,13,12], which turns a possibility measure $\Pi$ into a probability measure $P$, which is the center of gravity of $\mathcal{M}(\Pi)$. The subjective transformation is defined here for a finite universe $\Omega$ by

Definition 6. Let $\kappa$ be a probability distribution defined on $\Omega$. The possibility distribution $\pi_{[\kappa]}$, defined on $\Omega$, obtained by the subjective transformation of $\kappa$, is defined by

$$
\forall \omega \in \Omega, \quad \pi_{[\kappa]}(\omega)=\int_{\Omega} \min (\kappa(x), \kappa(\omega)) \mathrm{d} x .
$$

It is clear, when observing expression (17), that $\pi_{[\kappa]}(\omega)$ equals the hatched area $(a)+(b)$ of Fig. 6 with $(a)=\pi_{\leftarrow \kappa}(\omega)$ and $(b)=\lambda\left(I_{\omega}\right) \kappa(\omega)$. The difference at $\omega$ between $\pi_{[\kappa]}(\omega)$ and $\pi_{\leftarrow \kappa}(\omega)$ is $(b)=\lambda\left(I_{\omega}\right) \kappa(\omega)$ and is positive. Thus, the transformation of Dubois and Prade results in a more specific possibility distribution than the subjective transformation, indeed $\forall \omega \in \Omega, \pi_{[\kappa]}(\omega) \geqslant \pi_{\leftarrow \kappa}(\omega)$.

Theorem 7. Let $\kappa$ be a probability distribution defined on $\Omega$. The possibility distribution $\pi_{[\kappa]}$, obtained by the subjective transformation, is given for every $\omega \in \Omega$, by

$$
\pi_{[\kappa]}(\omega)=\pi_{\leftarrow \kappa}(\omega)+\lambda\left(I_{\omega}\right) \kappa(\omega) .
$$

Table 1 is a non-exhaustive list of symmetric summative kernels, commonly used in signal processing applications, associated with their transformed maxitive kernels.

The first column contains function $u$, which is the negative part of the basic summative kernel $\kappa$. While $\kappa$ is even:

$$
\kappa(\omega)=u(-|\omega|)
$$


Table 1

Common kernel transformations

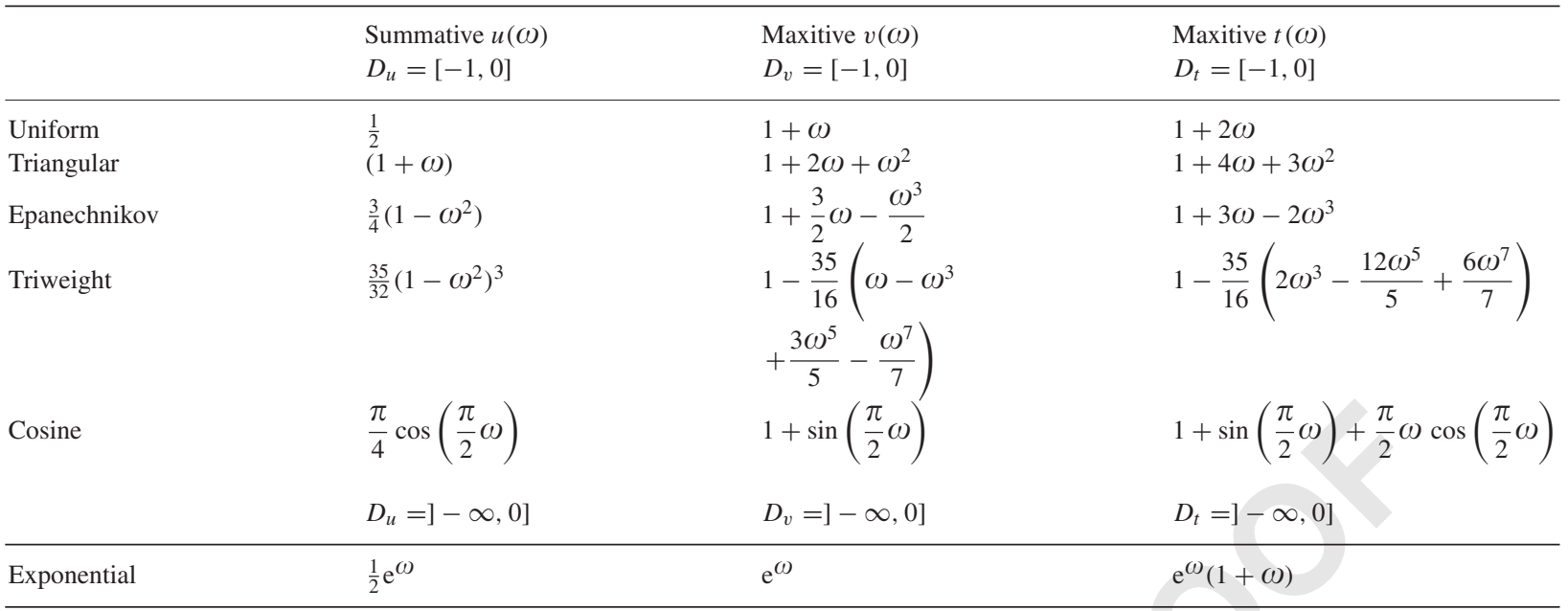

1 The second column contains function $v$, which is the negative part of the maxitive kernel $\pi_{\leftarrow \kappa}$. With $\kappa$ being even, $\pi_{\leftarrow \kappa}$ is even and therefore:

$$
\pi_{\leftarrow \kappa}(\omega)=v(-|\omega|) .
$$

The third column contains function $t$, which is the negative part of the maxitive kernel $\pi_{[\kappa]}$. With $\kappa$ being even, $\pi_{[\kappa]}$ is even and therefore:

$$
\pi_{[\kappa]}(\omega)=t(-|\omega|) .
$$

7 From Table 1, we can also retrieve $\kappa_{\Delta}$ and its associated maxitive kernels $\pi_{\leftarrow \kappa_{\Delta}}$ and $\pi_{\left[\kappa_{\Delta}\right]}$ :

$$
\begin{aligned}
& \kappa_{\Delta}(\omega)=\frac{1}{\Delta} u\left(-\frac{|\omega|}{\Delta}\right)=\frac{1}{\Delta} \kappa\left(\frac{\omega}{\Delta}\right), \\
& \pi_{\leftarrow \kappa_{\Delta}}(\omega)=v\left(-\left|\frac{\omega}{\Delta}\right|\right)=\pi_{\leftarrow \kappa}\left(\frac{\omega}{\Delta}\right), \\
& \pi_{\left[\kappa_{\Delta}\right]}(\omega)=t\left(-\left|\frac{\omega}{\Delta}\right|\right)=\pi_{[\kappa]}\left(\frac{\omega}{\Delta}\right) .
\end{aligned}
$$

The summative kernel, $\kappa_{\Delta}^{m}$, whose mode is on $\omega_{m}$, and its transformed maxitive kernels $\pi_{\leftarrow \kappa_{\Delta}^{m}}$ and $\pi_{\left[\kappa_{\Delta}^{m}\right]}$, are given by

$$
\begin{aligned}
& \kappa_{\Delta}^{m}(\omega)=\frac{1}{\Delta} u\left(-\frac{\left|\omega-\omega_{m}\right|}{\Delta}\right), \\
& \pi_{\leftarrow \kappa_{\Delta}^{m}}(\omega)=v\left(-\frac{\left|\omega-\omega_{m}\right|}{\Delta}\right), \\
& \pi_{\left[\kappa_{\Delta}^{m}\right]}(\omega)=t\left(-\frac{\left|\omega-\omega_{m}\right|}{\Delta}\right) .
\end{aligned}
$$

\subsection{Granularity}

The key idea, in defining granularity as a non-specificity index for a summative kernel $\kappa$, is the conjecture that the cardinality of $\pi_{\leftarrow \kappa}$ reflects the non-specificity of $\kappa$. In the converse sense, the specificity of probability distribution $\kappa$

13 can be quantified by measuring the specificity of $\pi_{\leftarrow \kappa}$.

In order to justify this conjecture, consider Fig. 7. For a given level $\alpha$, the alternatives $x$, with weights of specificity $15 \pi_{\leftarrow \kappa}(x)$ greater than $\alpha$, form $I_{\alpha}$, the $\alpha$-cut of $\pi_{\leftarrow \kappa}:\left\{x \in \Omega / \pi_{\leftarrow \kappa}(x) \geqslant \alpha\right\}$. Because of the definition of $\pi_{\leftarrow \kappa}$, such a 


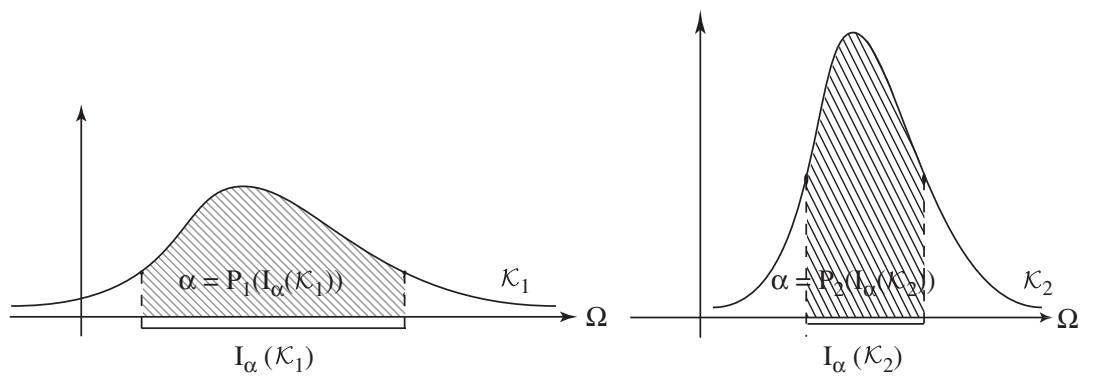

Fig. 7.

1 set can be identified with a set of the form given by expression (14): $\forall \alpha, \exists \omega / I_{\alpha}=I_{\omega}=\left\{x \in \Omega / \pi_{\leftarrow \kappa}(x) \geqslant \alpha\right\}=$ $\left\{x \in \Omega / P\left(I_{x}\right) \leqslant 1-\alpha\right\}$. The non-specificity index we propose is based on the idea that a summative kernel $\kappa_{2}$ is more 3 specific than a summative kernel $\kappa_{1}$, if most of the $I_{\alpha}\left(\kappa_{2}\right)$ are smaller than the $I_{\alpha}\left(\kappa_{1}\right)$. Since the $I_{\alpha}(\kappa)$ are the $\alpha$-cuts of $\pi_{\leftarrow \kappa}$, it is natural to measure the global specificity of $\kappa$ by using a specificity measure of $\pi_{\leftarrow \kappa}$. This constructive

5 characterization of the specificity of a summative kernel leads us to define our index of non-specificity, that we call granularity, in the following way:

7 Definition 8. Let $\kappa$ be a summative kernel. The granularity of $\kappa$, noted $\Gamma(\kappa)$, equals the cardinality of the possibility distribution $\pi_{\leftarrow \kappa}$ :

$$
\Gamma(\kappa)=\operatorname{card}\left(\pi_{\leftarrow \kappa}\right) .
$$

This implies an ordering on probability distributions:

11 Definition 9. Let $\kappa_{1}$ and $\kappa_{2}$ be summative kernels defined on $\Omega$, then $\kappa_{1}$ is said to be more granulated than $\kappa_{2}$ in the broad sense if and only if $\Gamma\left(\kappa_{1}\right) \geqslant \Gamma\left(\kappa_{2}\right)$, and strictly more granulated if and only if $\Gamma\left(\kappa_{1}\right)>\Gamma\left(\kappa_{2}\right)$.

13 Note that this index is a continuous counterpart of an information index, studied by Dubois and Hüllermeier [7] and proposed by Birnbaum [1] under the name of peakedness.

Beyond justifying the use of this index by intuitive considerations, Dubois and Hüllermeier [7] proved Theorem 10 that binds the peakedness index to the Shannon entropy of a probability vector $p=\left(p_{i}\right)_{i=1, \ldots,|\Omega|}$. Let us define $\Lambda_{\phi}(\cdot)$ as a generalized entropy given by

$$
\Lambda_{\phi}(p)=\sum_{i=1}^{|\Omega|} \phi\left(p_{i}\right),
$$

19 where the function $x \mapsto \phi(x)$ is strictly concave on $(0,1)$. Note that the function $x \mapsto-x \log (x)$ is strictly concave, which warrants the use of the term generalized entropy, since the Shannon entropy is defined by $H(p)=$ $21-\sum_{i=1}^{n} p_{i} \log \left(p_{i}\right)$,

Theorem 10. If a probability vector $p$ is less peaked than a probability vector $q$, then $\Lambda_{\phi}(p) \geqslant \Lambda_{\phi}(q)$, and if strictly less peaked, then $\Lambda_{\phi}(p)>\Lambda_{\phi}(q)$.

In other words, the ordering based on the entropy is a refinement of the relative peakedness ordering.

These remarks, as transposed to continuous probabilities and restricted to the Shannon entropy, bring some meaning to the definition of granularity. Indeed, it could be translated by the following conjecture:

27 Conjecture 11. If a summative kernel $\kappa_{1}$ is more granulated than a summative kernel $\kappa_{2}$, then $H\left(\kappa_{1}\right) \geqslant H\left(\kappa_{2}\right)$, and, if strictly more granulated, then $H\left(\kappa_{1}\right)>H\left(\kappa_{2}\right)$. 


\section{4. Adaptation of summative kernels in signal and image processing}

\subsection{Summative kernels in signal and image processing}

In signal processing, one key procedure is the modeling of the acquisition of a real continuous signal by a sensor. One of the most common ways of performing this modeling is to associate a summative kernel with the impulse response of the sensor. As a converse problem, a wide range of signal analysis procedures inherently rely on the existence of methods for reconstructing a continuous signal from a set of uniformly sampled corrupted values obtained by acquiring a signal. This reconstruction procedure can also be achieved by the use of a summative kernel.

Most of the summative kernels used in signal processing are monomodal, symmetric, bounded and separable. For a

9 summative kernel defined on an $p$-dimensional space $\Omega$, separable means that it can be decomposed into a product of $p$ summative kernels defined on the $p$ marginal spaces. This is typically used to reduce the complexity of kernel-based

11 algorithms. Table 2 offers a non-exhaustive list of such common kernels.

\subsubsection{Sampling, acquisition modeling}

13 Ideally, sensor acquisition at a location $\omega$ is modeled by a rectangular or crisp window which computes the average signal in a neighborhood of $\omega$ with radius $\Delta$. Note that $\Delta$ can be considered as the bandwidth of an averaging summative kernel $r_{\Delta}=1 / \Delta 1_{[-\Delta / 2, \Delta / 2]}$. Thus, $r_{\Delta}$ is assumed to be the impulse response of the sensor. The ideal measured signal, noted $S$ and defined on $\Omega$, is obtained by convolving the physical signal $s$ with this averaging filter $r_{\Delta}$,

$$
S(\omega)=\int_{\Omega} s(u) r_{\Delta}(\omega-u) \mathrm{d} u=\frac{1}{\Delta} \int_{\omega-\Delta / 2}^{\omega+\Delta / 2} s(u) \mathrm{d} u .
$$

This assumption of an averaging summative kernel is obviously naive, since such devices or sensors are seldom found in nature. Generally speaking, a sensor can be assimilated with an accumulator, that stores the information, or signal $s$, in a neighborhood of $\omega$ with radius $\Delta$, thus providing the accumulation of the signal, i.e. a signal $S$ proportional to the average of the signal $s$ at $\omega$ over $\Delta$. However, no real sensor works in this way, while physical accumulation generally involves capacity effects which tend to smooth its impulse response.

23 Thus, a relaxed or generalized model is usually preferred, consisting of replacing the averaging summative kernel $r_{\Delta}$ by a smoother summative kernel $v_{\Delta}$, which is more in accordance with sensor modeling. The role of the summative

25 kernel is to model the absorption of information contained in the underlying continuous physical signal around a given location. Then expression (30) becomes:

$$
S(\omega)=\int_{\Omega} s(u) v_{\Delta}(\omega-u) \mathrm{d} u=\left(s * v_{\Delta}\right)(\omega) .
$$

According to this model, a discrete signal can be seen as the values of the observed signal $S$ at regularly (or possibly

irregularly) distributed locations $\left(\omega_{i}\right)_{i=1, \ldots, n}$ of $\Omega$. We will restrict ourselves to the usual regularly distributed sampling, i.e. $\omega_{i}=\omega_{1}+\mathrm{i} h$, where $h$ is the sampling step.

Table 2

Comparison between the entropy and the granularity of common kernels

\begin{tabular}{llll}
\hline & $\kappa(\omega)$ & $h_{\kappa}$ & $\gamma_{\kappa}$ \\
\hline Triweight & $\frac{35}{32}\left(1-\omega^{2}\right)^{3}$ & 0.3086 & 0.5469 \\
Triangular over quadratic & $\frac{1}{\pi / 2-\ln 2}\left(\frac{1-|\omega|}{1+\omega^{2}}\right)$ & 0.4220 & 0.6015 \\
Triangular & $1-|\omega|$ & $\frac{1}{2}$ & 0.6667 \\
Cosine & $\frac{\pi}{4} \cos \left(\frac{\pi}{2} \omega\right)$ & 0.5484 & 0.7268 \\
Epanechnikov & $\frac{3}{4}\left(1-\omega^{2}\right)$ & 0.5680 & 0.75 \\
Trapezial & $\frac{2}{3}\left|\frac{1}{|\omega|<\frac{1}{2}}+\frac{4}{3}(1-|\omega|) 1\right| \omega \mid \geqslant \frac{1}{2}$ & 0.5721 & 0.7778 \\
Uniform & $\frac{1}{2} 1 \frac{1}{2}$ & 0.6931 & 1.5948 \\
Gaussian & $\frac{1}{\sqrt{2 \pi}} \mathrm{e}^{-\frac{\omega^{2}}{2}}$ & 1.4189 & 1.6931 \\
Exponential & $\frac{1}{2} \mathrm{e}^{-|\omega|}$ & 2 \\
\hline
\end{tabular}


1 Definition 12. A sampled signal consists of $n$ measurements of an original continuous signal $s$ at locations $\left(\omega_{i}\right)_{i=1, \ldots, n}$, given by $S_{i}=S\left(\omega_{i}\right)$.

\subsubsection{Reconstruction, interpolation}

Reconstruction can be presented as a reverse process, consisting of reconstructing a continuous signal, noted $\hat{S}$, from 5 its discrete observations $\left(S_{i}\right)_{i=1, \ldots, n}$.

Such a reconstruction mechanism can also be achieved by the use of summative kernels. In this case, the role of the summative kernel is to disperse or spread information contained in discrete observations, or measures, around them. Let $\eta_{\Delta}$ be the interpolation or reconstruction summative kernel with bandwidth $\Delta$.

9 Definition 13. Reconstruction of the continuous signal $\hat{S}$ from the sampled signal $\left(S_{i}\right)_{i=1, \ldots, n}$, which is defined at the locations $\left(\omega_{i}\right)_{i=1, \ldots, n}$ of $\Omega$, is given, for all $\omega$ in $\Omega$, by

$$
\hat{S}(\omega)=\sum_{i=1}^{n} \eta_{\Delta}\left(\omega-\omega_{i}\right) S_{i}=\left(\eta_{\Delta} * S\right)(\omega) .
$$

This reconstruction is an interpolation if $\forall i \in\{1, \ldots, n\}, \hat{S}\left(\omega_{i}\right)=S_{i}$.

\subsection{What is the adaptation of summative kernels?}

The adaptation between two summative kernels $\kappa_{\Delta_{1}}^{1}$ and $\kappa_{\Delta_{2}}^{2}$, consists of objectively choosing the bandwidth $\Delta_{2}$ of summative kernel $\kappa_{\Delta_{1}}^{1}$ in the same application. It generally consists of finding a relation between $\kappa^{1}, \Delta_{1}$ and $\kappa^{2}, \Delta_{2}$,

expressing a distance between the behaviors of $\kappa_{\Delta_{1}}^{1}$ and $\kappa_{\Delta_{2}}^{2}$, and deriving the adaptation relation from this relation, i.e.

$$
\Delta_{1}=f\left(\kappa^{1}, \kappa^{2}, \Delta_{2}\right) \quad \text { or } \quad \Delta_{2}=g\left(\kappa^{1}, \kappa^{2}, \Delta_{1}\right) .
$$

Two different approaches can be considered depending on the meaning of what is called the "behavior" of a summative kernel in a given application. The first adaptation methodology consists of identifying behavior indices of the summative

21 kernels. The second consists of optimizing the asymptotic behavior of Parzen Rosenblatt density estimators, which use the summative kernels involved in the adaptation. This simultaneous optimality gives rise to an explicit relation between the estimators, enabling the adaption of their asymptotic behavior.

\subsection{Granularity adaptation}

The granularity adaptation between two summative kernels $\kappa_{1}$ and $\kappa_{2}$ is based on the equalizing of their granularities. The principle of this method is simple and intuitive: as the granularity index of a kernel reflects its non-resolution power (i.e. its ability to collect or spread information), the equalizing of the granularities of two kernels leads to a very natural adaptation of their behavior in applications where they can be seen as weighted neighborhoods ensuring smooth interplay between continuous and discrete domains.

The construction of an adaptation relation of the form (33), involving granularity, requires a preliminary theorem. Let $\gamma_{\kappa}$ be the granularity of the basic summative kernel $\kappa$, i.e. $\gamma_{\kappa}=\Gamma\left(\kappa_{1}\right)$.

Theorem 14. For any summative kernel $\kappa_{\Delta}$,

$$
\Gamma\left(\kappa_{\Delta}\right)=\Delta \gamma_{\kappa}
$$

Proof 15. $\Gamma\left(\kappa_{\Delta}\right)=\int_{\Omega} \pi_{\leftarrow \kappa_{\Delta}}(\omega) \mathrm{d} \omega=\int_{\Omega} \pi_{\leftarrow \kappa_{1}}(\omega / \Delta) \mathrm{d} \omega$, see expression (23). Then, $\Gamma\left(\kappa_{\Delta}\right)=\int_{\Omega} \Delta \pi_{\leftarrow \kappa_{1}}(x) \mathrm{d} x=$ $\Delta \gamma_{\kappa}$. 


\section{ARTICLE IN PRESS}

1 As previously mentioned, we say that two kernels $\kappa_{\Delta_{1}}^{1}$ and $\kappa_{\Delta_{2}}^{2}$ are granularity-adapted if $\Gamma\left(\kappa_{\Delta_{1}}^{1}\right)=\Gamma\left(\kappa_{\Delta_{2}}^{2}\right)$. Because of Theorem 14, this equation leads to $\Delta_{1} \gamma_{\kappa^{1}}=\Delta_{2} \gamma_{\kappa^{2}}$ and therefore:

$$
\frac{\Delta_{2}}{\Delta_{1}}=\frac{\gamma_{\kappa^{1}}}{\gamma_{\kappa^{2}}}=\xi_{\kappa^{1}}^{\kappa^{2}}
$$

The granularity adaptation relation is

$$
\Delta_{2}=\xi_{\kappa^{1}}^{\kappa^{2}} \Delta_{1},
$$

$\xi_{\kappa^{1}}^{\kappa^{2}}$ is called the granularity adaptation coefficient between $\kappa^{1}$ and $\kappa^{2}$. Kernel $\kappa_{\Delta_{1}}^{1}$ is said to be granularity adapted to $7 \quad$ kernel $\kappa_{\Delta_{2}}^{2}$ if $\Delta_{2}=\xi_{\kappa^{1}}^{\kappa^{2}} \Delta_{1}$.

The granularity adaptation coefficient simply requires values of the basic granularities $\gamma_{\kappa^{1}}$ and $\gamma_{\kappa^{2}}$ of the summative

9 kernels $\kappa^{1}$ and $\kappa^{2}$. Table 2 offers a list of the granularities of common basic summative kernels, thus facilitating the recovery of the granularity adaptation coefficient. For example, the adaptation coefficient between the triangular kernel

$11 T$ and the Epanechnikov kernel $E$ is given by $\xi_{T}^{E}=\gamma_{T} / \gamma_{E}=\frac{8}{9}$.

\subsection{Entropy adaptation}

13 Shannon entropy adaptation is based on the identification of the Shannon entropies of summative kernels $\kappa^{1}$ and $\kappa^{2}$ involved in the adaptation. This is exactly the same principle as that of granularity adaptation.

15 The construction of an adaptation relation of the form (33), involving the Shannon entropy, requires a preliminary theorem. Let $h_{\kappa}$ be the Shannon entropy of the basic summative kernel $\kappa$, i.e. $h_{\kappa}=H\left(\kappa_{1}\right)$.

Theorem 16. For any summative kernel $\kappa_{\Delta}$,

$$
H\left(\kappa_{\Delta}\right)=\log (\Delta)+h_{\kappa} .
$$

19 Proof 17. $H\left(\kappa_{\Delta}\right)=-\int_{\Omega} \kappa_{\Delta}(\omega) \log \left(\kappa_{\Delta}(\omega)\right) \mathrm{d} \omega=-\int_{\Omega}(1 / \Delta) \kappa_{1}(\omega / \Delta) \log \left((1 / \Delta) \kappa_{1}(\omega / \Delta)\right) \mathrm{d} \omega=-\int_{\Omega} \kappa_{1}(x)$ $\left(\log (1 / \Delta)+\log \left(\kappa_{1}(x)\right)\right) \mathrm{d} x=\log (\Delta) \int_{\Omega} \kappa_{1}(x) \mathrm{d} x-\int_{\Omega} \kappa_{1}(x) \log \left(\kappa_{1}(x)\right) \mathrm{d} x=\log (\Delta)+h_{\kappa}$.

21 As previously mentioned, we say that two kernels $\kappa_{\Delta_{1}}^{1}$ and $\kappa_{\Delta_{2}}^{2}$ are entropy adapted if $H\left(\kappa_{\Delta_{1}}^{1}\right)=H\left(\kappa_{\Delta_{2}}^{2}\right)$. Because of Theorem 16, this equation leads to $\log \left(\Delta_{1}\right)+h_{\kappa^{1}}=\log \left(\Delta_{2}\right)+h_{\kappa^{2}}$ and therefore:

$$
\frac{\Delta_{2}}{\Delta_{1}}=\mathrm{e}^{h_{\kappa^{1}}-h_{\kappa^{2}}}=\phi_{\kappa^{1}}^{\kappa^{2}}
$$

The entropy adaptation relation is

$$
\Delta_{2}=\phi_{\kappa^{1}}^{\kappa^{2}} \Delta_{1}
$$

$\phi_{\kappa^{1}}^{\kappa^{2}}$ is called the entropy adaptation coefficient between $\kappa^{1}$ and $\kappa^{2}$. Kernel $\kappa_{\Delta_{1}}^{1}$ is said to be entropy adapted to kernel

$27 \kappa_{\Delta_{2}}^{2}$ if $\Delta_{2}=\phi_{\kappa^{1}}^{\kappa^{2}} \Delta_{1}$.

The entropy adaptation coefficient simply requires values of the basic entropies $h_{\kappa^{1}}$ and $h_{\kappa^{2}}$ of the summative kernels

$29 \kappa^{1}$ and $\kappa^{2}$. Table 2 offers a list of the entropies of common basic summative kernels, thus facilitating the recovery of the entropy adaptation coefficient. For example, the entropy adaptation coefficient between the triangular kernel $T$ and

31 the Epanechnikov kernel $E$ is given by $\phi_{T}^{E}=\mathrm{e}^{h_{T}-h_{E}}=0.9343$.

\subsection{AMISE adaptation}

The AMISE approach [30] belongs to the second family of adaptations. In fact, the ability of a summative kernel to collect information is used in the so-called Parzen Rosenblatt $[20,25]$ density estimator $f_{\kappa \Delta}$. The probability density 
1 function $f$, describing a random variable $X$ on $\Omega$, is estimated by using a sample of $N$ independent and identically distributed observations $\left(X_{1}, \ldots, X_{N}\right)$ of $X$, and a summative kernel $\kappa_{\Delta}$.

$$
f_{\kappa \Delta}(\omega)=\frac{1}{N \Delta} \sum_{i=1}^{N} \kappa\left(\frac{\omega-X_{i}}{\Delta}\right)=\frac{1}{N} \sum_{i=1}^{N} \kappa_{\Delta}\left(\omega-X_{i}\right) .
$$

The asymptotic behavior of the Parzen Rosenblatt density estimator can be characterized by an upper bound of the $L_{2}$-error (also called the Mean Integrated Squared Error) between the density estimator $f_{\kappa} \Delta$ and the underlying density function $f$ of the random variable $X$, given by MISE $=\left\|f-f_{\kappa \Delta}\right\|_{L_{2}}=\int_{\Omega}\left(f(\omega)-f_{\kappa \Delta}(\omega)\right)^{2} \mathrm{~d} \omega$. The upper bound of this $L_{2}$-error, called the Asymptotic Mean Integrated Squared Error (in the case of the Parzen Rosenblatt estimator $f_{\kappa \Delta}$ ) is analytically given by: $\operatorname{AMISE}\left(f_{\kappa \Delta}\right)=R(\kappa) / N \Delta+\Delta^{4} \sigma_{\kappa}^{4} R\left(f^{\prime \prime}\right) / 4$, with $R(\phi)=\int \phi^{2}(u) \mathrm{d} u$ and $\sigma_{\phi}^{2}=\int u^{2} \phi(u) \mathrm{d} u$.

Next, as proved in $[22,27,29]$, the bandwidth, $\Delta^{*}$, which minimizes the AMISE is given by

$$
\Delta^{*}=\left(\frac{R(\kappa)}{\sigma_{\kappa}^{4}}\right)^{1 / 5}\left(R\left(f^{\prime \prime}\right) N\right)^{-1 / 5} .
$$

11 Note that there is no one-to-one correspondence between $\kappa$ and its associated optimal bandwidth $\Delta^{*}$. Indeed, $\Delta^{*}$ depends not only on $f$, the density to estimate and $N$, the number of observations, but also on $\kappa$, the kernel chosen for 13 the estimation.

The AMISE adaptation involves computation of the ratio of the two optimal bandwidths $\Delta_{1}^{*}$ and $\Delta_{2}^{*}$, obtained in the same estimation conditions (i.e. the same underlying function $f$ and the same number of observations $N$ ) from $\kappa^{1}$ and $\kappa^{2}$. This ratio is noted as

$$
\zeta_{\kappa^{1}}^{\kappa^{2}}=\frac{\Delta_{2}^{*}}{\Delta_{1}^{*}}=\left(\frac{R\left(\kappa^{2}\right)}{\sigma_{\kappa^{2}}^{4}}\right)^{1 / 5}\left(\frac{R\left(\kappa^{1}\right)}{\sigma_{\kappa^{1}}^{4}}\right)^{-1 / 5},
$$

which depends neither on $f$ nor on $N$.

The AMISE adaptation relation is

$$
\Delta_{2}=\zeta_{\kappa^{1}}^{\kappa^{2}} \Delta_{1}
$$

$21 \zeta_{\kappa^{1}}^{\kappa^{2}}$ is called the AMISE adaptation coefficient between $\kappa^{1}$ and $\kappa^{2}$. Kernel $\kappa_{\Delta_{1}}^{1}$ is said to be AMISE adapted to kernel $\kappa_{\Delta_{2}}^{2}$ if $\Delta_{2}=\zeta_{\kappa^{1}}^{\kappa^{2}} \Delta_{1}$.

23 This is, however, a shortcoming of this method which is applied to the whole bandwidth range, whereas it is fully valid only for the optimal bandwidth.

\subsection{Comparisons of adaptation coefficients and indices}

Table 2 presents a comparison of the granularity index $\gamma_{\kappa}$ and the Shannon entropy index $h_{\kappa}$ for nine common continuous summative kernels $\kappa$. The entropy indices are sorted by increasing order. This order is the same as the granularity index order. This suggests that Theorem 10 is also verified for continuous probability distributions. Table 3 presents the adaptation coefficients of three summative kernels to the uniform kernel. The second column presents the AMISE adaptation coefficients [30], computed with expression (42). The third column presents the granularity adaptation coefficients computed with expression (35). The fourth column presents the entropy adaptation coefficients computed with expression (38). The three adaptation coefficients are quite close and seem to go in the same direction, i.e. $\zeta_{\mathrm{U}}^{\kappa^{1}}>\zeta_{\mathrm{U}}^{\kappa^{2}} \Leftrightarrow \xi_{\mathrm{U}}^{\kappa^{1}}>\xi_{\mathrm{U}}^{\kappa^{2}} \Leftrightarrow \phi_{\mathrm{U}}^{\kappa^{1}}>\phi_{\mathrm{U}}^{\kappa^{2}}$. In the next section, we propose experiments based on these adaptations to highlight the fact that granularity properly measures the non-resolution power of the summative kernels used in common signal processing applications.

\section{Experiments}

We carried out numerous experiments on applications using the summative kernels: filtering, impulse response modeling, image decimation. We present here some results concerning image filtering and sensor modeling. 
Table 3

Comparison between AMISE, granularity and entropy adaptation coefficients

\begin{tabular}{llll}
\hline & Granularity $\xi_{\mathrm{U}}^{\kappa}$ & Entropy $\phi_{\mathrm{U}}^{\kappa}$ & AMISE $\zeta_{\mathrm{U}}^{\kappa}$ \\
\hline Epanechnikov: $\frac{3}{4}\left(1-x^{2}\right) \mathbb{1}_{[-1,1]}$ & 1.3333 & 1.1333 & 1.2724 \\
Triweight: $\frac{35}{32}\left(1-x^{2}\right)^{3} \mathbb{1}_{[-1,1]}$ & 1.8286 & 1.4689 & 1.7115 \\
Gaussian: $\frac{1}{\sqrt{2 \pi}} \mathrm{e}^{-\frac{x^{2}}{2}}$ & 0.6270 & 0.4839 & 0.5747 \\
\hline
\end{tabular}

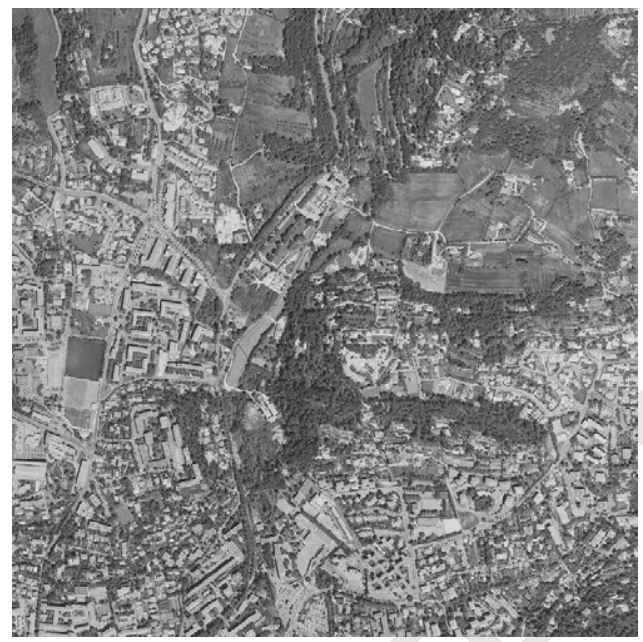

Fig. 8. Bouches-du-Rhône.

\section{5.1. Experimental comparison between adaptation methods in signal filtering}

This experiment concerns adaptation comparison in a filtering context. As an illustrative experiment, we propose to filter a $3200 \times 3200$ satellite image using four different summative kernels: Gaussian, Uniform, Epanechnikov and Triweight. This satellite image, depicted in Fig. 8, was provided by the Institut Géographique National (IGN) and represents a part of the Bouches-du-Rhône. The sampling step is taken as a unit, i.e. $h=1$.

The images resulting from the filtering are compared when the bandwidths of the four summative kernels are adapted with AMISE, entropy and granularity adaptations. The comparison has also been effected with no adaptation. Fig. 9 shows the mean of the $L_{2}$-distances between the filtered images for different values of the bandwidth of the uniform kernel. The values of the bandwidths of the other summative kernels are obtained using the adaptation relations (36), (39) and (43). The comparison was performed on the central part of the filtered images to avoid side effects.

11 As a preliminary trivial remark, any adaptation is better than no adaptation, whatever the chosen method. Using the worst adaptation method will, on average, provide a mean $L_{2}$-distance that is 20 times smaller than the mean

$13 \quad L_{2}$-distance obtained without adaptation. This result is not shown in Fig. 9, because it could lower the informativity of the plotting.

15 When the bandwidths are quite small $\left(\Delta_{\mathrm{U}} \leqslant 10\right)$, the filtered images are not smooth enough for the filters to provide equivalent results (see Figs. 10-12). In this case, adaptation with AMISE provides a better result (i.e. the images are closer) than granularity adaptation. Conversely, when the adapted bandwidth exceeds this value, the filtering is strong enough to clear the main details from the satellite image (see Figs. 13-15), and the dissimilarities between the

19 different results are stabilized. In this last case, granularity adaptation provides a better result than AMISE adaptation. Therefore, since filtering consists of removing details, granularity adaptation seems to be more appropriate in this kind 21 of application.

Now, when comparing granularity adaptation to Shannon adaptation, the same remarks are valid with a lower 23 threshold $\left(\Delta_{\mathrm{U}}<4\right)$. Therefore, granularity adaptation seems to be more appropriate than entropy adaptation in image 


\section{ARTICLE IN PRESS}

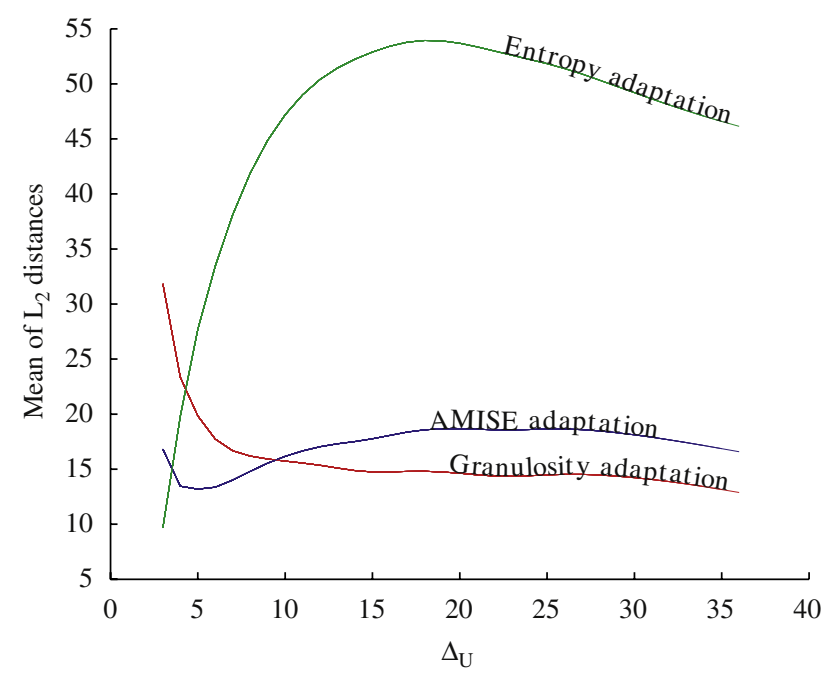

Fig. 9. Comparison of the efficiency of adaptation methods in image filtering.

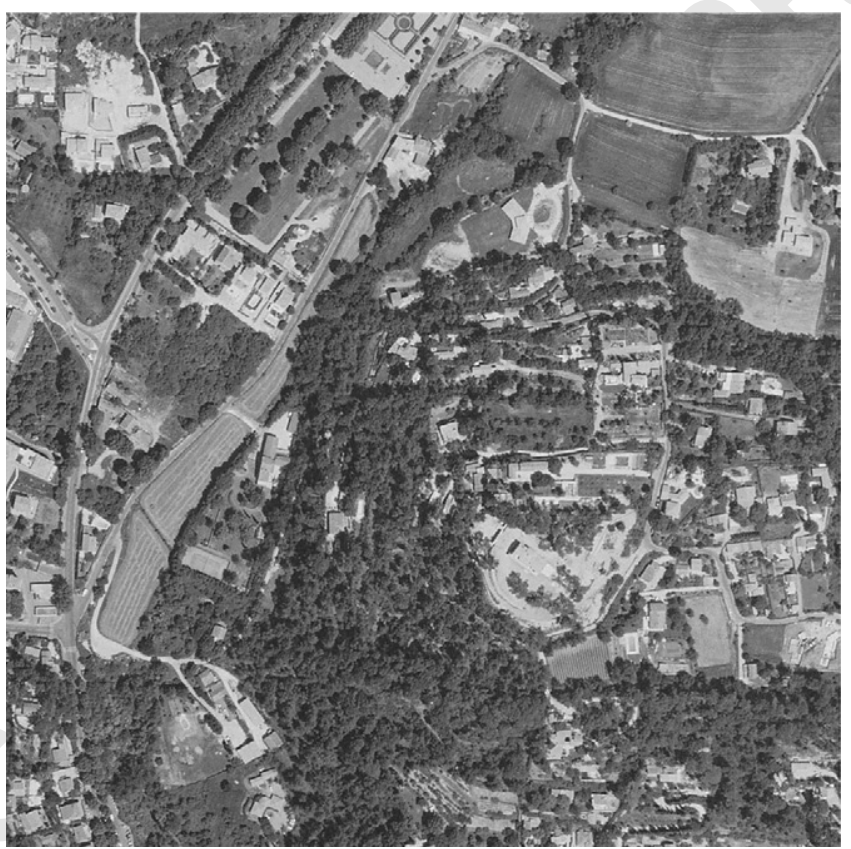

Fig. 10. $\Delta_{\mathrm{U}}=1$.

1 filtering. Actually, entropy adaptation does not seem to be a proper adaptation method in such a context: the distance between the filtered adapted images tends to increase as $\Delta$ increases.

3 Note that, in this kind of discrete filtering application, the spreading and collecting properties of a summative kernel can hardly be differentiated. In fact, the filtered value of a discrete signal at a given location with a given kernel can be

5 thought of either as the spreading, with this kernel, of all the sampled values evaluated at the considered location, or as the collecting of all sampled values in the weighted neighborhood defined by the kernel at the considered location.

7 Therefore, regarding filtering application, the spreading and collecting abilities of a given summative kernel can be sought as dual properties. 


\section{ARTICLE IN PRESS}

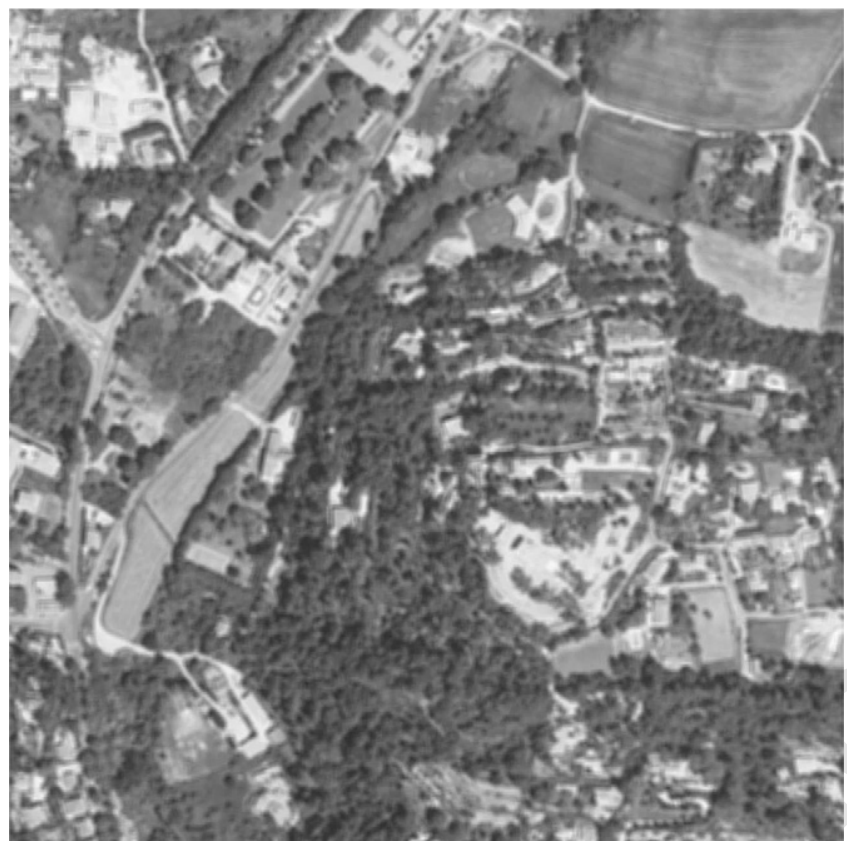

Fig. 11. $\Delta_{\mathrm{U}}=5$.

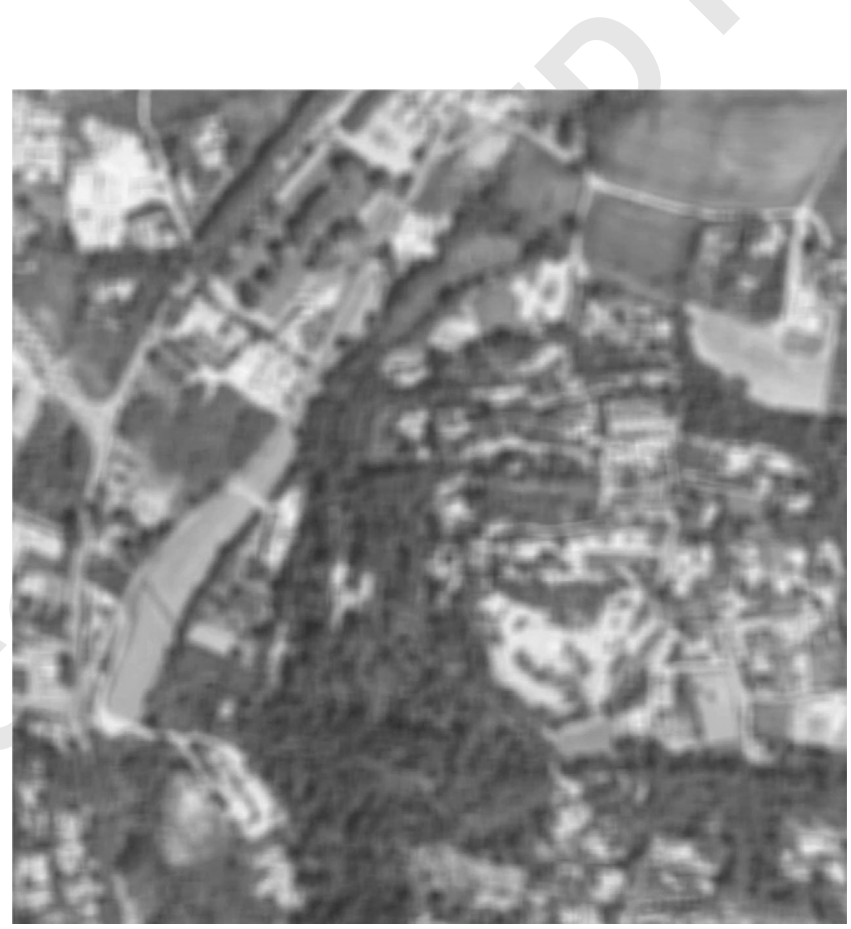

Fig. 12. $\Delta_{\mathrm{U}}=10$.

In this kind of application, the number of sampled values that the filter absorbs or spreads depends on the bandwidth of the summative kernel. Remember that the sampling step is $h=1$. A uniform kernel-based filter, with a bandwidth of 5 , will act on $11 \times 11$ pixels. More generally, a uniform kernel-based filter, with a bandwidth $\Delta_{\mathrm{U}}$, will act on $2 \Delta_{\mathrm{U}}+1 \times 2 \Delta_{\mathrm{U}}+1$ pixels. Therefore, the uniform filter acts on sets of pixels whose cardinality increases with the 


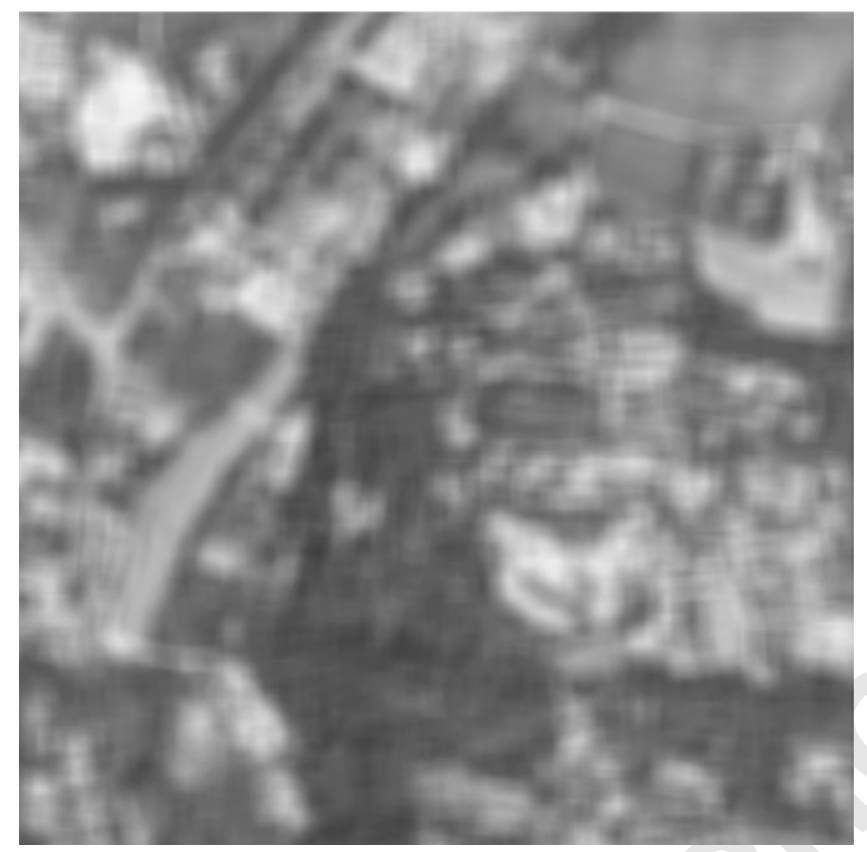

Fig. 13. $\Delta_{\mathrm{U}}=20$.

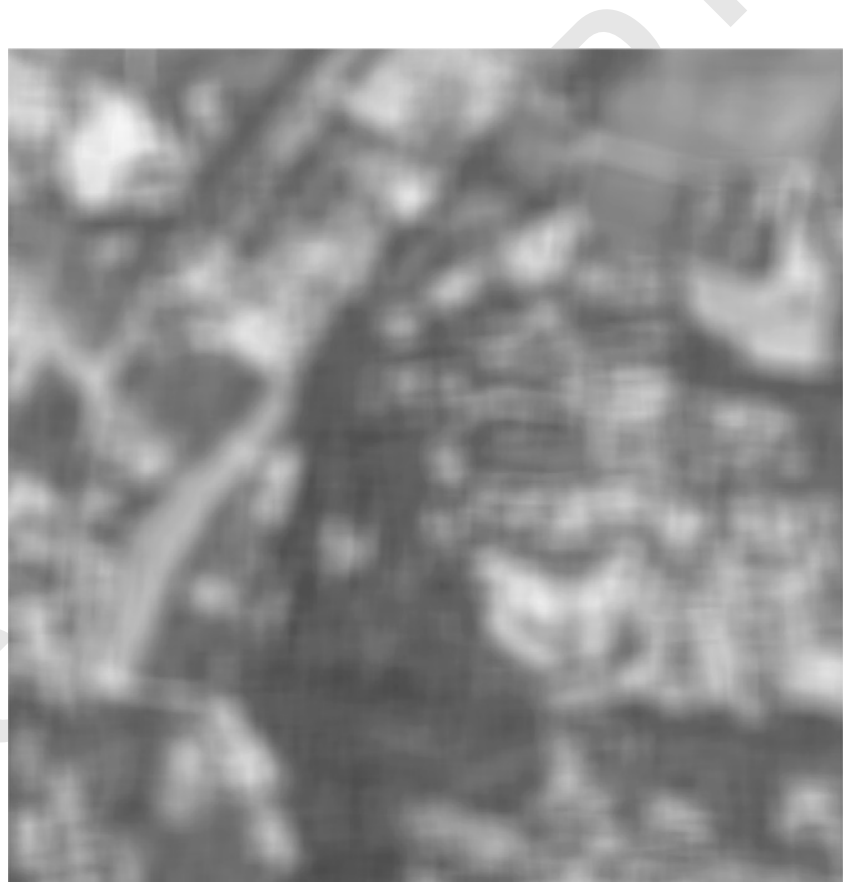

Fig. 14. $\Delta_{\mathrm{U}}=25$.

1 bandwidth. This property is also consistent with other summative kernels. Since, for large bandwidths, granularity adaptation is better than other adaptation methods, the granularity index can be seen as a good marker of the absorption or spreading abilities of a summative kernel, and therefore is a good index of non-resolution power.

Figs. 10-15 represent filtered images of the satellite image depicted in Fig. 8, obtained with the uniform kernel whose bandwidth is taken from $\Delta_{\mathrm{U}}=1$ to $\Delta_{\mathrm{U}}=30$. 


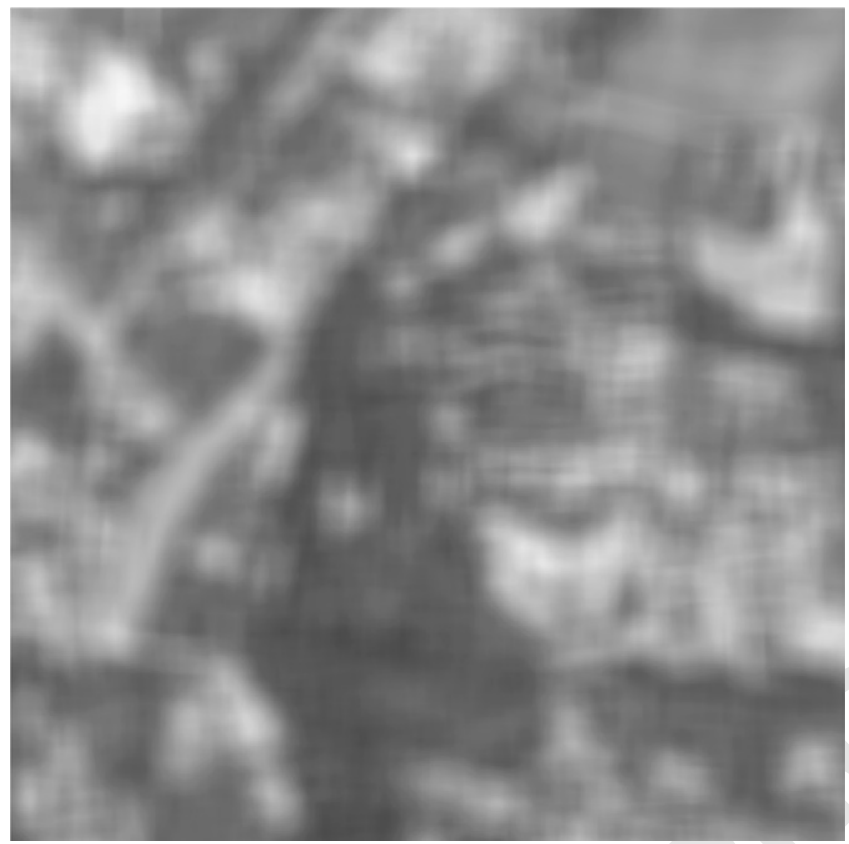

Fig. 15. $\Delta_{U}=30$.

\subsection{Experimental comparison between adaptation methods in continuous discrete interplay}

The property we aim to highlight, within this experiment, is the ability of the granularity index to characterize the absorption capability of a summative kernel. This experiment consists of simulating the behavior of one detector of a nuclear imaging device [14]. Such a detector is designed to count the photons emitted in a certain direction during a given period. A complete device is made up of several detectors. Each one is associated with a collecting direction. The proportion of photons detected by a sensor over the complete number of detected photons is the signature of the density of radioactivity in the direction associated with the sensor. The density of radioactivity detected in a certain direction is known to be ruled by a Poisson process, and depends not only on the measured density of radioactivity but also on $N$, the total number of detected photons, and on $\kappa$, the impulse response of the sensor.

In this experiment, the radioactive zone is supposed to be approximately punctual, which means that it is assumed to be symmetrically distributed. We perform this experiment by modeling this radioactive zone by a centered uniform distribution on $[-50,50]$. We suppose that all the photons emitted by this active zone are detected by the device, and we focus on the density of photons detected by one of the detectors.

We compute the detected density when modeling the impulse response of this sensor by the four summative kernels used in the previous experiment: Uniform, Epanechnikov, Triweight and Gaussian, with adapted bandwidths. This computation is made for $\Delta_{\mathrm{U}}$ ranging from 0 to 20 and for a number of emitted photons ranging from 1 to 1000 .

We compute the $L_{2}$-distance between the densities obtained with the four presented summative kernels when using different adaptation methods and, also, with no adaptation. Then, we compute the mean of the $L_{2}$-distances obtained over 100 different experimental data sets.

As a first remark, whatever $\Delta_{\mathrm{U}}$ and $N$, granularity adaptation is better than no adaptation. Fig. 16 shows the difference, noted $d_{\mathrm{NG}}$, between the average distance with no adaptation and the average distance with granularity adaptation. The results obtained for the other adaptation methods are similar, and therefore not plotted here.

Fig. 17 shows the difference, noted $d_{\mathrm{AG}}$, between the average distance with AMISE adaptation and the average distance with granularity adaptation. For most of the parameters $\Delta_{\mathrm{U}}$ and $N$, the granularity adaptation is better than the AMISE adaptation method, except for some pairs $\left(N, \Delta_{\mathrm{U}}\right)$, with small $N$ and small $\Delta_{\mathrm{U}}$.

Fig. 18 shows the difference, noted $d_{\mathrm{EG}}$, between the average distance with entropy adaptation and the average distance with granularity adaptation. Whatever $\Delta_{\mathrm{U}}$ and $N$, the granularity adaptation is better than the entropy adaptation method. 


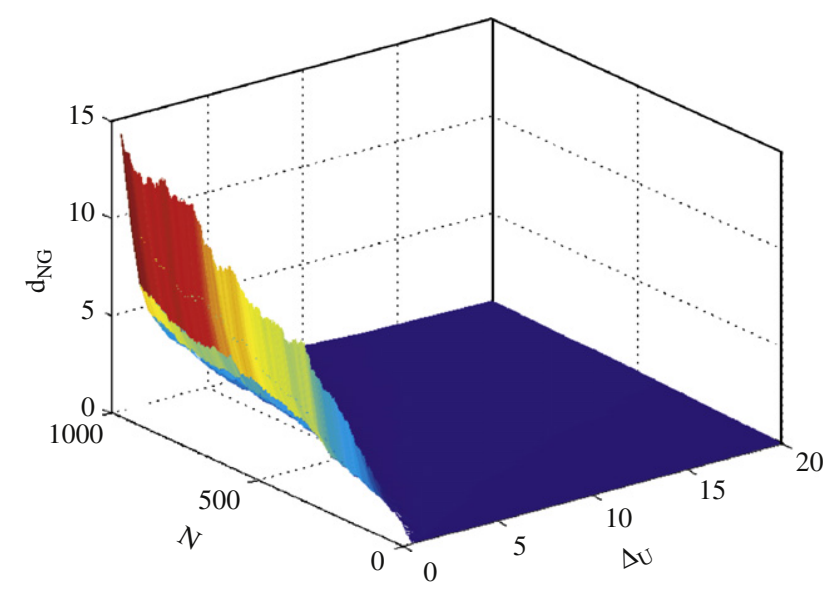

Fig. 16. Comparison between no adaptation and granularity adaptation.

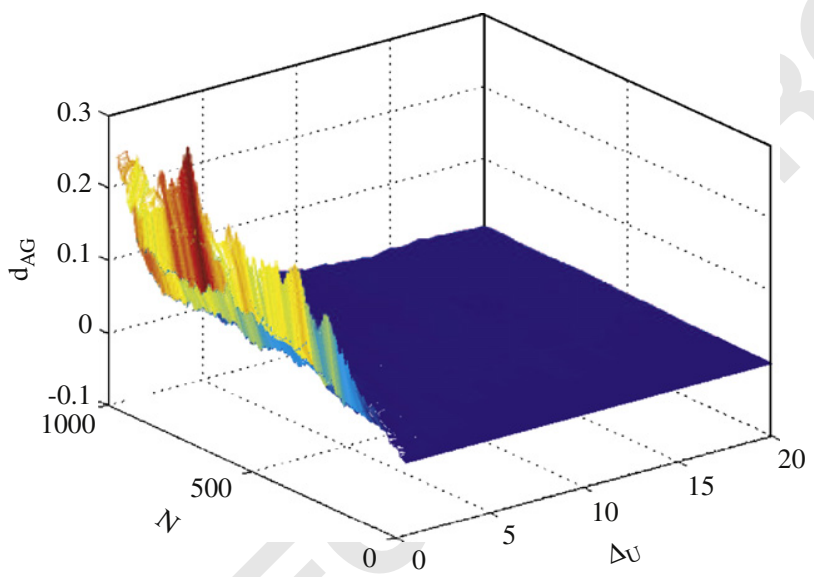

Fig. 17. Comparison between AMISE and granularity adaptations.

1 Since this experiment uses the absorption property of the summative kernels, we can conclude that the granularity better captures the ability of a summative kernel to absorb information than the entropy does.

\section{Conclusion}

In a digital signal processing context, summative kernels are widely used either to model the impulse response of a sensor, or to ensure a smooth interplay between continuous and discrete domains. In such a context, it is of practical importance to be able to use an index that characterizes the behavior of a summative kernel in different applications.

When a kernel is used to model the impulse response of a sensor, one of the characteristics of practical importance to be derived from the kernel is the resolution power of the sensor. This resolution power is usually defined as being inversely proportional to the minimal distance between two measured features that can be separated by using the measured signal. When modeling the measurement by a uniform kernel defined on an interval, this minimal distance is directly proportional to the length of the interval. Since this length is, by definition, the granularity of a uniform kernel, it seems straightforward to conjecture that the granularity of any summative kernel associated with the impulse response of a sensor is a marker of its non-resolution power. Granularity can thus be seen as an index reflecting non-resolution power, i.e. the ability of a summative kernel to spread or collect information in the usual signal processing applications. Note that the Shannon entropy index is not consistent with this definition of a non-resolution power index, since the Shannon entropy index of a uniform kernel defined on an interval tends to $-\infty$ as its length tends to 0 . 


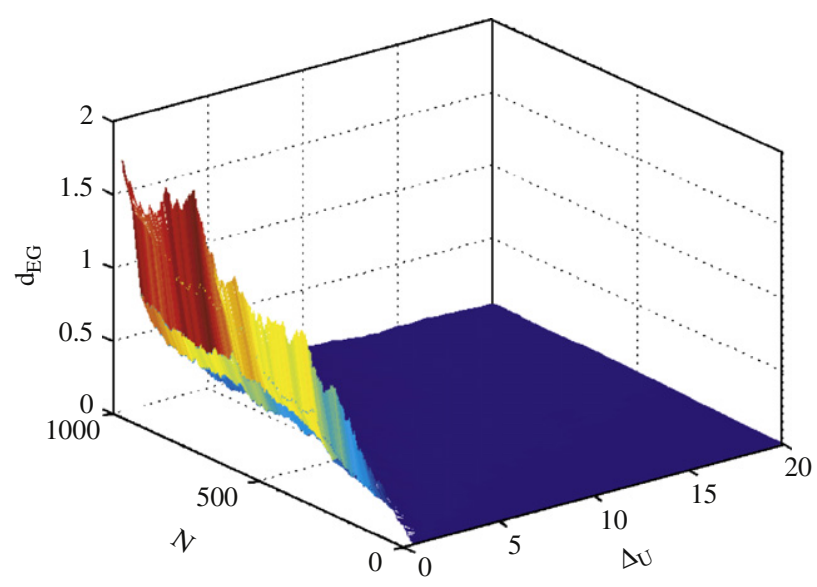

Fig. 18. Comparison between entropy and granularity adaptations.

A key step in our definition of granularity is to conjecture that the (non-)resolution power of a summative kernel and the (non-)specificity of a probability distribution are dual properties. In fact, we define the granularity of a summative kernel as an index characterizing its non-specificity, considering this summative kernel as being a probability distribution. This specificity is the ability, for a probability distribution, to be concentrated on a set of minimal Lebesgue measure. Birnbaum [1], by means of the peakedness index, defined a non-specificity measure that has been studied by Dubois and Hüllermeier [7]. It has been observed that the construction of the peakedness is based on the probability/possibility transformation of Dubois and Prade [12]. It appears that granularity is simply the peakedness index of Birnbaum, defined for continuous probability distributions.

9 The numerous experiments we carried on highlighted the fact that, when two summative kernels with different shapes have the same granularity, their behavior in typical kernel-based signal processing applications are quite close, and,

11 moreover, closer than two summative kernels having the same Shannon index. For adaptation purposes, the AMISE adaptation method derived from the Parzen-Rosenblatt kernel-based density estimation may also be considered. In fact,

13 in applications where few data are involved, this adaptation sometimes seems to work better than the granularity-based adaptation. However, even if the AMISE adaptation can be useful in comparing summative kernels, it seems difficult to derive a direct, useful, non-resolution index from the adaptation coefficients. It should still be an interesting research track to follow.

17 Among numerous possible uses, our new index could also yield new criteria for finding the optimal shape of a summative kernel for a particular application. Moreover, due to the separability property of Theorem 14, the use of such criteria could lead to very simple methods, and to computationally low-cost algorithms. One could also consider directly inferring the non-resolution power of a sensor by applying the probability/possibility transformation directly to the experimental data collected when the sensor is measuring a known pattern. Finally, since a maxitive kernel can be viewed as representing the family of the summative kernels with lower or equal granularity, it sounds sensible to consider a new way of performing signal processing based on replacing summative kernels by maxitive kernels in the usual applications.

\section{References}

[1] Z.W. Birnbaum, On random variables with comparable peakedness, Ann. of Math. Statist. 19 (1948) 76-81.

[2] S. Bodjanova, Granulation of a fuzzy set: nonspecificity, Inform. Sci. 177 (2007) 4430-4444.

[3] J. Canny, A computational approach to edge detection, in: IEEE Trans. on Pattern Analysis and Machine Intelligence, Vol. 8, 1986, pp. 679-698.

[4] G. De Cooman, D. Aeyels, Supremum-preserving upper probabilities, Inform. Sci. 118 (1999) 173-212.

[5] R. Deriche, Using Canny's criteria to derive a recursively implemented optimal edge detector, Internat. J. Computer Vision 1 (2) (1987) $167-187$.

[6] D. Dubois, Possibility theory and statistical reasoning, Comput. Statist. Data Anal. 51 (2006) 47-69.

33 [7] D. Dubois, E. Hüllermeier, Comparing probability measures using possibility theory: a notion of relative peakedness, Internat. J. Approx. Reason. (2006). 
1 [8] D. Dubois, H. Prade, Théorie des possibilités: applications à la représentation des connaissances en informatique, Masson, 1988.

[9] D. Dubois, H. Prade, When upper probabilities are possibility measures, Fuzzy Sets and Systems 49 (1992) 65-74.

3 [10] D. Dubois, H. Prade, Fuzzy sets and probability: misunderstandings, bridges and gaps, in: Proc. Second IEEE Conf. on Fuzzy Systems, 1993, pp. 1059-1068.

5 [11] D. Dubois, H. Prade, L. Foulloy, G. Mauris, Probability-possibility transformations, triangular fuzzy sets, and probabilistic inequalities, Reliable Computing 10 (2004) 273-297.

7 [12] D. Dubois, H. Prade, S. Sandri, On possibility/probability transformations, in: R. Lowen, M. Roubens (Eds.), Fuzzy Logic. State of the Art, Kluwer Academic Publishers, Dordrecht, 1993, pp. 103-112.

9 [13] D. Dubois, H. Prade, P. Smets, New semantics for quantitative possibility theory, in: Internat. Symp. on Imprecise Probabilities and Their Applications, Ithaca, USA, 2001.

11 [14] R. Formiconi, A. Pupi, A. Passeri, Compensation of spatial system response in spect with conjugate gradient reconstruction technique, Phys. Med. Biol. 34 (1) (1989) 69-84.

[15] F. Jacquey, K. Loquin, F. Comby, O. Strauss, Non-additive approach for gradient-based edge detection, in: ICIP07, Internat. Conf. on Image Processing, 2007.

15 [16] J. Jan, Digital Signal Filtering, Analysis and Restoration, IET, 2000.

[17] G.J. Klir, M.J. Wierman, Uncertainty Based Information: Elements of Generalized Information Theory, Physica, 1998.

17 [18] D. Morales, L. Pardo, I. Vajda, Uncertainty of discrete stochastic systems: general theory and statistical inference, IEEE Trans. Systems Man Uncertainty—Part A: Systems and Humans 26 (6) (1996) 681-697.

19 [19] H.T. Nguyen, Some mathematical tools for linguistic probabilities, Fuzzy Sets and Systems 2 (1979) 53-65.

[20] E. Parzen, On estimation of a probability density function and mode, Ann. of Math. Statist. 33 (1962) 1065-1076.

21 [21] Z. Pawlak, Rough Sets: Theoretical Aspects of Reasoning about Data, Kluwer Academic Publishers, Dordrecht, 1991.

[22] B.L.S. Prakasa Rao, Nonparametric Functional Estimation, Academic Press, New York, 1983.

23 [23] A. Rényi, On the measures of entropy and information, in: 4th Berkeley Symp. on Mathematical Statistics and Probabilities, Vol. 1, 1961, pp. 547-561.

25 [24] A. Rényi, On the foundations of information theory, Rev. Internat. Statist. Inst. 33 (1965) 1-14.

[25] M. Rosenblatt, Remarks on some nonparametric estimates of a density function, Ann. of Math. Statist. 27 (1956) $832-837$.

27 [26] L. Schwartz, Théorie des distributions, Vol. 1, Hermann, Paris, 1950.

[27] D.W. Scott, Multivariate Density Estimation, Wiley/Interscience, New York, 1992.

29 [28] C.E. Shannon, A mathematical theory of communication, The Bell System Technical Journal 27 (1948) $379-423$.

[29] B.W. Silvermann, Density Estimation for Statistics and Data Analysis, Monographs on Statistics and Applied Probability, Vol. 26, Chapman \& Hall, London, 1986.

[30] J.S. Simonoff, Smoothing Methods in Statistics, Springer, Berlin, 1996.

33 [31] Ph. Smets, R. Kennes, The transferable belief model, Artificial Intelligence 66 (1994) 191-234.

[32] M. Unser, Splines: a perfect fit for signal and image processing, IEEE Signal Process. Mag. 16 (1999) 22-38.

35 [33] P. Walley, Statistical Reasoning with Imprecise Probabilities, Chapman \& Hall, London, 1991.

[34] P. Walley, Towards a unified theory of imprecise probability, Internat. J. Approx. Reason. 24 (2-3) (2000) 125-148.

37 [35] R.R. Yager, Entropy and specificity in a mathematical theory of evidence, Internat. J. General Systems 9 (1983) 249-260.

[36] L.A. Zadeh, Fuzzy sets as a basis for a theory of possibility, Fuzzy Sets and Systems 1 (1978) 3-28. 